\title{
Synthesis of New Fluorine/Phosphorus Substituted 6-(2'-Amino Phenyl)-3-Thioxo- 1,2,4-Triazin-5(2H, 4H)One and Their Related Alkylated Systems as Molluscicidal Agent as against the Snails Responsible for Bilharziasis Diseases
}

\author{
Abeer N. Al-Romaizan*, Mohammed S. T. Makki, Reda M. Abdel-Rahman \\ Department of Chemistry, Faculty of Science, King Abdul Aziz University, \\ Jeddah, KSA \\ Email: ar-orkied@hotmail.com
}

Received 22 April 2014; revised 5 June 2014; accepted 22 June 2014

Copyright (C) 2014 by authors and Scientific Research Publishing Inc.

This work is licensed under the Creative Commons Attribution International License (CC BY). http://creativecommons.org/licenses/by/4.0/

(c) (i) Open Access

\begin{abstract}
New fluorine substituted 6-(5'-fluoro-2'-triphenylphosphiniminophenyl) 3-thioxo-1,2,4-triazin-5 $(2 \mathrm{H}, 4 \mathrm{H})$ one (2) was obtained via Wittig's reaction of the corresponding 6-(5'-fluoro-2'-aminophenyl)-3-thioxo-1,2,4-triazinone (1). Behavior of compound 2 towards alkylating agents and/or oxidizing agents was studied were, $N$-hydroxyl $(3)$, Mannich base $(4,5), S$-alkyl $(6,7,8)$ and thiazolo $[3,2-b][1,2,4]$ triazinones $(10-14)$ and or 3-disulfide (18), 3-sulfonic acid 19 and 1,2,4-triazin-3,5Dionne (20) derivatives obtained. Structures of the new products are established by elemental and spectral data. The new targets obtained screened as Molluscicidalagents against Biomophlaria Alexandrina snails responsible for Bilharziasis diseases, in compare with Baylucide as standard drug.
\end{abstract}

\section{Keywords}

Fluorine, Phosphorus, Sulfur-1,2,4-Triazine, Characteristic Properties, Molluscicidal Activity

\footnotetext{
"Corresponding author.

How to cite this paper: Al-Romaizan, A.N., et al. (2014) Synthesis of New Fluorine/Phosphorus Substituted 6-(2'-Amino Phenyl)-3-Thioxo-1,2,4-Triazin-5(2H, 4H)One and Their Related Alkylated Systems as Molluscicidal Agent as against the Snails Responsible for Bilharziasis Diseases. International Journal of Organic Chemistry, 4, 154-167. http://dx.doi.org/10.4236/ijoc.2014.42017
} 


\section{Introduction}

The incorporation of fluorine atoms into a heterocyclic nitrogen molecule frequently provides properties of pharmacological interest as compared to their non-fluorinated analogs [1]-[5]. Also, bonded phosphorus atoms with $\mathrm{S}, \mathrm{O}, \mathrm{N}$ and $\mathrm{C}$-atoms of heterocyclic systems enhance their important properties as herbicides, pesticides and insecticides [6]-[11]. On the other hand, 3-thioxo-1,2,4-triazin-5-one derivatives and their N-and S-alkyl derivatives have gained considerable attention due to their well as medicinal utility such as anti-HIV, anti AIDS and anticancer agents [12]-[16]. Literature reveals that no reports of a molecular scaffold containing these important cores. With this based upon these observations. The present work aims to synthesis and chemical reactivity of 1,2,4-triazinone bearing, fluorine, phosphorus and sulfur atoms through alkylation reactions and the new systems as Molluscicidal agents against Biomophalaria Alexandrina snails by removal from the wastewater (Clean water).

\section{Experimental}

Melting points were determined with an electro-thermal Bibbly Stuart Scientific Melting point SMPI (UK). A Perkin Elmer (Lambda EZ-210) double beam spectrophotometer (190 - $1100 \mathrm{~nm}$ ) used for recording the electronic spectra. A Perkin Elmer model RXI-FT-IR 55,529 $\mathrm{cm}^{-1}$ used for recording the IR spectra (EtOH as solvents). A Brucker advance DPX $400 \mathrm{MHz}$ using TMS as an internal standard for recording the ${ }^{1} \mathrm{H} /{ }^{13} \mathrm{C} \mathrm{NMR}$ spectra in deuterated DMSO ( $\delta$ in ppm). AGC-MS-QP 1000 Ex model is used for recording the mass spectra. Hexafluorobenzene was used as external standard for ${ }^{19} \mathrm{~F}$ NMR at $8425 \mathrm{MHz}$ and ${ }^{31} \mathrm{P}$ (in $\mathrm{CDCl}_{3}, 101.25 \mathrm{MHz}$ ) [17]. Elemental analysis was performed on Micro Analytical Center of National Reaches Center-Dokki, Cairo, Egypt. Compound $\mathbf{1}$ was prepared according the reported method [14] and compound $\mathbf{1 5}$ as procedure published [18].

\section{6-(5’-Fluor phenyl)2'amino-3-thioxo-1,2,4-triazine-5(2H, 4H)one (1)}

Equimolar mixture of 5-fluoroisatin (in $100 \mathrm{ml} \mathrm{NaOH}, 5 \%$ ) and thiosemicarbazide (in $10 \mathrm{ml} \mathrm{H}_{2} \mathrm{O}$ ) reflux for 2 $\mathrm{h}$, then cold and poured onto ice-HCl. The solid result was filtered off and crystallized from EtOH as yellow crystals to give 1. Yield (80\%), m.p. $263^{\circ} \mathrm{C}-265^{\circ} \mathrm{C}$. Analytical data, Found $\mathrm{C}=44.91, \mathrm{H}=2.90, \mathrm{~F}=7.58, \mathrm{~N}=$ 23.40, $\mathrm{S}=13.29 \%$; Calculated for $\mathrm{C}_{9} \mathrm{H}_{7} \mathrm{FN}_{4} \mathrm{OS}$ (238) $\mathrm{C}=45.37, \mathrm{H}=2.94, \mathrm{~F}=7.98 \mathrm{~N}=23.52, \mathrm{~S}=13.44 \%, \mathrm{M} / \mathrm{Z}$ (256, $\mathrm{M}+\mathrm{H}_{2} \mathrm{O}, 5 \%$ ), base peak (68, 100\%), 148 (21), 136 (18), 110 (30), 96 (50), 82 (58), 70 (78); UV: ( $\lambda_{\max }$ EtOH) $280 \mathrm{~nm}$. IR vcm ${ }^{-1}=3424\left(\mathrm{NH}_{2}\right)$ 3258, $3169(\mathrm{NH}, \mathrm{NH}), 1685(\mathrm{C}=\mathrm{O}), 1618\left(\mathrm{NH}_{2}\right), 1545(\mathrm{C}=\mathrm{N}), 1263$ (C-F): 858, 818 (aryl CH) $685(\mathrm{C}-\mathrm{F}){ }^{1} \mathrm{H}$ NMR $(\mathrm{DMSO})=14.66,16.66,10.90$ (each 1H, s, 3NH), 8.68 - 8.06, 7.69 - 7.64, 7.39, $7.39-7.30$ (3H, aryl protons) ${ }^{13} \mathrm{C}$ NMR $=\delta 179.47(\mathrm{C}=\mathrm{S}), 162(\mathrm{C}=\mathrm{O}), 159$ - 157 (spin coupling $\mathrm{C}-\mathrm{F}), 138.54(\mathrm{C}=\mathrm{N}), 131.82,121.8,121.51$ (aromatic carbons), 78.14, 77.71 (C5-C6 1,2,4-triazine).

\section{6-[5'-Fluoro-2'(triphenylphosphinimino)phenyl]-3-thioxo-1,2,4-triazine-5(2H, 4H)one (2)}

A mixture of 1 (0.01 mol) and triphenyl phosphine $(0.01 \mathrm{~mol})$ in acetonitrile $(20 \mathrm{ml})$, THF (20 ml) reflux for 2 h then cold. The solid produced and crystallized from EtOH to give 2 as deep yellowish crystals. Yield (70\%) m.p. $249^{\circ} \mathrm{C}-250^{\circ} \mathrm{C}$. Analytical date Found $\mathrm{C}=64.60, \mathrm{H}=3.96, \mathrm{~F}=3.70, \mathrm{~N}=11.01, \mathrm{~S}=6.33 \%$. Calculated for $\mathrm{C}_{27} \mathrm{H}_{20} \mathrm{FN}_{4} \mathrm{OPS}$ (498): $\mathrm{C}=65.06, \mathrm{H}=4.01, \mathrm{~F}=3.81, \mathrm{~N}=11.24, \mathrm{~S}=6.42 \%, \mathrm{M} / \mathrm{Z}$ (498.00) 370 (4), 290 (10), 171 (60), 159 (100), 128 (20), 118 (40), 102 (65), 92 (58), 76 (58), 65 (40); UV: ( $\lambda_{\max }$ EtOH) 310 nm. IR vcm ${ }^{-1}$ = $3335(\mathrm{NH}), 1658(\mathrm{C}=\mathrm{O}), 1380(\mathrm{P}=\mathrm{N}), 1250(\mathrm{C}-\mathrm{F}), 1087$ (C-S) 1045, (P-N) 879 (aryl CH), $650(\mathrm{C}-\mathrm{F}) .{ }^{1} \mathrm{H}$ NMR (DMSO): 14.56, 12.78 (each 1H, s, 2NH), 8.21, 7.76, 7.66, 7.65, 7.65, 7.64, 7.484, 7.840, 7.47, 7.464, 7.460, 7.45, 7.398, 7.391, 7.38, 7.37, 7.29, 7.28, 7.28, 7.27, 7.02, 7.01, 7.009, 7.005, 6.994, 6.990, 6.866, 6.859, 6.852, 6.845, (18H of aromatic protons). ${ }^{13} \mathrm{C}$ NMR (DMSO) $=\delta 179.74(\mathrm{C}=\mathrm{S}), 163.0(\mathrm{C}=\mathrm{O}), 138.62(\mathrm{C}-\mathrm{F}), 131(\mathrm{C}=\mathrm{N})$, 118.94 - 107.94 (aromatic carbons), 7.76, $77.21\left(\mathrm{C}_{5}-\mathrm{C}_{6}\right.$ of 1,2,4-triazine).

2,4-Di(hydroxymethyl)-6-(5'-fluoro-2'-triphenylphosphiniminophenyl)-3-thioxo-1,2,4-triazine-5-one (3)

A mixture of $2(0.01 \mathrm{~mol})$ and formaldehyde $(0.02 \mathrm{~mol})$, in methanol $(50 \mathrm{ml})$ reflux for $2 \mathrm{~h}$, cold. The solid obtained filtered off and crystallized from MeOH to give 2 as faint yellow crystals. Yield $=(65 \%) \mathrm{m} . \mathrm{p} .280^{\circ} \mathrm{C}-$ $282^{\circ} \mathrm{C}$. Analytical date: Found $\mathrm{C}=61.92, \mathrm{H}=4.21, \mathrm{~F}=5.23, \mathrm{~N}=9.93, \mathrm{~S}=5.43 \%$ Calculated for $\mathrm{C}_{29} \mathrm{H}_{24} \mathrm{FN}_{4} \mathrm{PSO}_{3}$ (558): $\mathrm{C}=62.36, \mathrm{H}=4.30, \mathrm{~F}=3.40, \mathrm{~N}=10.03, \mathrm{~S}=5.73 \% . \mathrm{UV}:\left(\lambda_{\max } \mathrm{EtOH}\right)=363 \mathrm{~nm} . \mathrm{IR}$ $v \mathrm{~cm}^{-1}=3346$ (b, $\left.2 \mathrm{OH}\right)$ 2974, $2889\left(2 \mathrm{CH}_{2}\right), 1646(\mathrm{C}=\mathrm{O}), 1382(\mathrm{P}=\mathrm{N}), 1240(\mathrm{C}-\mathrm{F}), 1086(\mathrm{C}-\mathrm{S}), 1046(\mathrm{P}-\mathrm{N}), 879$ (aryl CH), 755 (C-F). ${ }^{1} \mathrm{H}$ NMR (DMSO) $\delta 8.34$ - 6.84 (18 aromatic protons), 4.8, 4.4 (each s, 2H, alcoholic 2OH) 2.62, 2.58 (each s, 4H, 2CH$)_{2}{ }^{13} \mathrm{C}$ NMR (DMSO) $=\delta 179.86-179.68(\mathrm{C}=\mathrm{S}), 163.07(\mathrm{C}=\mathrm{O}), 159.62,158.03$ (C-F), (C-N), $138.60(\mathrm{C}=\mathrm{N}), 132.121$ - 107.94 (aromatic carbons), 77.7577 .32 (C5-C6 of 1,2,4-triazine), 40.57 - 
40.46, $40.32-4.14\left(2 \mathrm{CH}_{2}\right)$.

2,4-Di(Piperidinomethyl)-6-(5'-fluoro-2'-triphenylphosphin-iminophenyl)-3-thioxo-1,2,-4-triazine-5-one (4)

A mixture of $2(0.01 \mathrm{~mol})$, piperidine $(0.02 \mathrm{~mol})$ and formaldehyde $(0.02 \mathrm{~mol})$ in methanol $(50 \mathrm{ml})$ reflux for $2 \mathrm{~h}$, cold. The solid produced filtered off and crystallized from $\mathrm{MeOH}$ to give 4 as yellow crystals. Yield $=(60 \%)$ m.p. $179^{\circ} \mathrm{C}-180^{\circ} \mathrm{C}$. Analytical date found $\mathrm{C}=67.41, \mathrm{H}=5.83, \mathrm{~F}=2.55, \mathrm{~N}=11.97, \mathrm{~S}=4.33 \%$. Calculated for $\mathrm{C}_{39} \mathrm{H}_{42} \mathrm{FN}_{6} \mathrm{OPS}$ (692): $\mathrm{C}=67.63, \mathrm{H}=12.13, \mathrm{~F}=2.74, \mathrm{~N}=12.13, \mathrm{~S}=4.62 \% . \mathrm{IR} v \mathrm{~cm}^{-1}=3062$ (aromatic $\mathrm{CH}$ ), 2936, 2840 (aliphatic $\left.\mathrm{CH}_{2}\right), 1721(\mathrm{C}=\mathrm{O}), 1538(\mathrm{C}=\mathrm{N}), 1468$ (deform $\left.\left.\mathrm{CH}_{2}\right), 1389\right)(\mathrm{P}=\mathrm{N}), 1248(\mathrm{C}-\mathrm{F}), 1184$ $(\mathrm{C}=\mathrm{S}), 1049$ (P-N), 885, 815, 754 (aryl CH), 709 (C-F). ${ }^{1} \mathrm{H}$ NMR (DMSO): $\delta 8.23$ - 6.80 (18H, aromatic), 2.95, 2.92, 2.89 and $2.58\left(\mathrm{CH}_{2}\right.$ of piperdine, $\left.\mathrm{N}-\mathrm{CH}_{2}-\mathrm{N}\right) .{ }^{13} \mathrm{C}$ NMR (DMSO) $=\delta 172(\mathrm{C}=\mathrm{S}), 154(\mathrm{C}=\mathrm{O}), 147.25(\mathrm{C}-\mathrm{F})$, $137.54(\mathrm{C}=\mathrm{N}), 116.10$ - 108 (aromatic carbons), 77.80, 77.39 ( $\mathrm{C}_{5}-\mathrm{C}_{6}$ of 1,2,4-triazine), 40 - 58, 40.47, 40.33, 40.19, $40.05\left(\mathrm{CH}_{2}\right.$ of piperidine) $39.91-39.63\left(\mathrm{~N}^{-} \mathrm{CH}_{2}-\mathrm{N}\right)$.

2,4-Di(4'-arylaminomethyl)-6-(5'-fluoro-2'-triphenylphosphiniminophenyl)-3-thioxo-1,2,4-triazine-5ones (5a \& 5b)

A mixture of 2 (0.01 mol ) and formaldehyde (0.02 mol), 4-fluoroaniline and/or 4-aminoantipyrine (0.02 mol) in methanol (50 ml) warm under reflux for $2 \mathrm{~h}$, then cold. The solid thus obtained, filtered off and crystallized from $\mathrm{EtOH}$ to give $\mathbf{5 a} \& \mathbf{5 b}$ as yellow crystals.

Compound 5a, yield (75\%) m.p. $210^{\circ} \mathrm{C}-212^{\circ} \mathrm{C}$. Analytical data: Found $\mathrm{C}=65.80, \mathrm{H}=4.11, \mathrm{~F}=7.47, \mathrm{~N}=$ 10.89, $\mathrm{S}=4.01 \%$. Calculated for $\mathrm{C}_{41} \mathrm{H}_{32} \mathrm{~F}_{3} \mathrm{~N}_{6} \mathrm{OPS}(744), \mathrm{C}=66.12, \mathrm{H}=4.30 ; \mathrm{F}=7.66 ; \mathrm{N}=11.29, \mathrm{~S}=4.30 \%$. $\mathrm{M} / \mathrm{Z}=744$ (M, 0, 0\%), 370 (1), 367 (25), 290 (60), 272 (30), 248 (20), 218 (42), 169 (100), 128 (85), 102 (100), 65 (100). UV: ( $\left.\lambda_{\max } \mathrm{EtOH}\right) 364 \mathrm{~nm}$; IR vcm ${ }^{-1}=3343(\operatorname{aryl}-\mathrm{NH}), 2974,2889\left(\mathrm{CH}_{2}\right), 1650(\mathrm{C}=\mathrm{O}), 1382(\mathrm{P}=\mathrm{N})$, 1250 (C-F), 1086 (C-S), 1045 (P-N) 879 (aryl CH). ${ }^{1} \mathrm{H}$ NMR (DMSO) $=\delta 12.71$ (s, 1H, NH), 12.55 (s, 1H, NH), 7.41, 7.39, 7.32, 7.06, 7.01, 6.99, 6.98, 6.97, 6.91, $6.90 \& 6.89,6.88,6.878,6.873,6.86 \& 6.85,6.84,6.83,6.77$ \& 6.768, 6.761, 6.754, 6.742, 6.735 (aromatic $\mathrm{CH}), 5.18-5.15$ \& $5.14-5.13\left(4 \mathrm{H}, \mathrm{CH}_{2}\right.$ of $\left.\mathrm{N} \mathrm{CH}_{2}-\mathrm{NH}\right) .{ }^{13} \mathrm{C}$ $\mathrm{NMR}=(\mathrm{DMSO})=\delta 178.0(\mathrm{C}=\mathrm{S}), 161.02(\mathrm{C}=\mathrm{O}) 144.99(\mathrm{C}-\mathrm{F}), 138.37(\mathrm{C}=\mathrm{N}), 130.65-114.11$ (aromatic car-

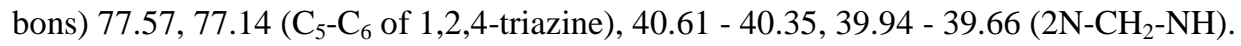

Compound 5b, yield $(60 \%)$; m.p. $200^{\circ} \mathrm{C}-202^{\circ} \mathrm{C}$. Analytical data: Found $\mathrm{C}=65.89, \mathrm{H}=4.91, \mathrm{~F}=2.04, \mathrm{~N}=$ 14.83, $\mathrm{S}=3.35 \%$; Calculated for $\mathrm{C}_{51} \mathrm{H}_{46} \mathrm{FN}_{10} \mathrm{O}_{3} \mathrm{PS}$ (928), $\mathrm{C}=65.94, \mathrm{H}=9.95, \mathrm{~F}=2.04, \mathrm{~N}=15.08, \mathrm{~S}=3.44 \%$. IR vcm ${ }^{-1}$ : 3277 (b, NH), 2974 (aliphatic $\left.\mathrm{CH}_{3}\right) 2840\left(\mathrm{CH}_{2}\right), 1697,1660(2 \mathrm{C}=\mathrm{O}), 1604(\mathrm{C}=\mathrm{N}), 1542(\mathrm{C}=\mathrm{N}), 1482$ (deform, $\mathrm{CH}_{2}$ ), $1416(\mathrm{P}=\mathrm{N}), 1370$ (NCSN), $1274(\mathrm{C}-\mathrm{F}), 1152(\mathrm{C}-\mathrm{S}), 1045(\mathrm{P}-\mathrm{N}), 878,810,750$ (aromatic $\mathrm{CH}$ ), 650 (C-F). ${ }^{1} \mathrm{H}$ NMR (DMSO) $\delta 7.48-7.26$ (aromatic CH) 5.07 (s, 1H, NH), 3.1 - 3.0 (s, 1H, NH), 2.99 - 2.88, $2.84-2.82\left(2 \mathrm{CH}_{2}\right), 2.62-2.41,2.34-2.23,2.229-2.2221,2.16-2.07(4 \mathrm{Me}) .{ }^{13} \mathrm{C}$ NMR $=(\mathrm{DMSO}) \delta 179.81$ $(\mathrm{C}=\mathrm{S}) 159.11(\mathrm{C}=\mathrm{O}), 142(\mathrm{C}-\mathrm{F}), 138.0(\mathrm{C}=\mathrm{N}), 129.38-123.31$ (aromatic carbons), 77.54, $77.11\left(\mathrm{C}_{5}-\mathrm{C}_{6}\right.$ of 1,2,4-

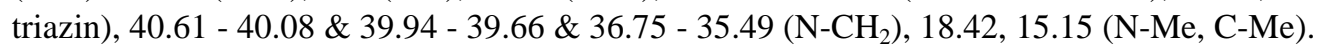

[6-(5'-Fluoro-2'-triphenylphosphiniminophenyl)-5-0xo-1,2,4-triazine-3-yl]thioacetic acid (6)

Equimolar mixture of 2 and monochloroacetic acid in DMF $(20 \mathrm{ml}$ ) warm for 30/min, then poured onto ice. The solid yielded filtered off and crystallized form EtOH to give $\mathbf{6}$ as faint yellow crystals. Yield (80\%), m.p. $187^{\circ} \mathrm{C}-188^{\circ} \mathrm{C}$. Analytical data: Found: $\mathrm{C}=62.42, \mathrm{H}=3.81, \mathrm{~F}=3.20, \mathrm{~N}=9.85, \mathrm{~S}=5.57 \%$; Calculated for $\mathrm{C}_{29} \mathrm{H}_{22} \mathrm{FN}_{4} \mathrm{O}_{3} \mathrm{PS}$ (556). $\mathrm{C}=62.58, \mathrm{H}=3.95, \mathrm{~F}=3.41, \mathrm{~N}=10.07, \mathrm{~S}=5.75 . \mathrm{IR} v \mathrm{~cm}^{-1}=3327$ (b, OH, NH), 2973, $2884\left(\mathrm{CH}_{2}\right), 1659$ (b, $\left.2 \mathrm{C}=\mathrm{O}\right), 1440$ (deform $\left.\mathrm{CH}_{2}\right), 1380(\mathrm{P}=\mathrm{N}), 1250(\mathrm{C}-\mathrm{F}), 1087$ (C-S), $1045(\mathrm{P}-\mathrm{N}) 880(\mathrm{Ar}$ $\mathrm{CH}), 810$ (Ar CH). ${ }^{1} \mathrm{H}$ NMR (DMSO) $\delta=10.31(\mathrm{~s}, 1 \mathrm{H}, \mathrm{NH}), 8.06,8.0,7.98$ - 7.97, 79.86 - 7.84, 7.73, \& 7.726, 7.721, 7.14, 7.08, 7.67 \& 7.66, 7.65, 7.63, 7.53 - 7.51, 7.48 - 7.35 \& 7.34, 6.997, 6.992, 6.838 - $6.824(18 \mathrm{CH}$, aromatic) \& $4.74(\mathrm{~s}, 1 \mathrm{H}, \mathrm{OH}$ of $\mathrm{COOH}), 3.86-3.24\left(2 \mathrm{H}, \mathrm{CH}_{2}\right) .{ }^{13} \mathrm{C}$ NMR: (DMSO): $\delta 168.29(\mathrm{C}=\mathrm{S}), 165.40$ $(\mathrm{C}=\mathrm{O}), 157.16(\mathrm{C}=\mathrm{O}), 142.13(\mathrm{C}-\mathrm{F}) 131.35(\mathrm{C}=\mathrm{N}), 130.04-102.25$ (aromatic carbons), 72.43, $72.22\left(\mathrm{C}_{5}-\mathrm{C}_{6}\right.$ of 1,2,4-traizine), $34.95-34.81\left(\mathrm{CH}_{2}\right.$ carbon).

1,1-Di[6-(5'-Fluoro-2'-triphenylphosphiniminophenyl)-5-oxo-1,2,-4-triazine-3'yl]dimercaptoacetic acid (7)

A mixture of $2(0.02 \mathrm{~mol})$ and 1,1-dicholoracetic acid $(0.01 \mathrm{~mol})$ in DMF $(20 \mathrm{ml})$ reflux for $30 \mathrm{~min}$, cold then poured into ice. The resulted solid filtered off and crystallized from dioxin to give $\mathbf{7}$ as faint yellow crystals, yield (60\%) m.p. $238^{\circ} \mathrm{C}-240^{\circ} \mathrm{C}$. Analytical data: Found $\mathrm{C}=63.45, \mathrm{H}=3.49, \mathrm{~F}=3.39, \mathrm{~N}=10.39, \mathrm{~S}=5.88 \%$, Calculated for $\mathrm{C}_{56} \mathrm{H}_{40} \mathrm{~F}_{2} \mathrm{~N}_{8} \mathrm{O}_{4} \mathrm{P}_{2} \mathrm{~S}_{2}(1052) \mathrm{C}=63.87, \mathrm{H}=3.80, \mathrm{~F}=3.61, \mathrm{~N}=10.64, \mathrm{~S}=6.08 \%$. IR vcm ${ }^{-1}=3425$, 3259, $3170(\mathrm{OH}, \mathrm{NH}, \mathrm{NH}), 1865,1680(\mathrm{C}=\mathrm{O}), 1618(\mathrm{C}=\mathrm{N}), 1476,1452$ (aliphatic $\mathrm{CH}), 1360(\mathrm{P}=\mathrm{N}), 1252$ (C-F), 1193 (C-S), 1045 (P-N), 903, 859, 818, 758 (aryl CH) 685 (C-F). ${ }^{1} \mathrm{H}$ NMR (DMSO): $\delta$ 12.79, 12.78 (each s, 2NH), 10.75 (s, 1H, OH), 8.21 - $6.84\left(18 \mathrm{CH}\right.$, aromatic), 2.82 - $2.59(\mathrm{~s}, 1 \mathrm{H}, \mathrm{CH}){ }^{13} \mathrm{C}$ NMR: $\delta 179.72(\mathrm{C}=\mathrm{S})$, 
163 (C=O), 159 (C-S), 158.0 (C-S), 138.61 (C-F), $132.18(\mathrm{C}=\mathrm{N}), 121.12$ - 107.94 (aromatic carbons), 77.66, $77.45\left(\mathrm{C}_{5}-\mathrm{C}_{6}\right)$ of 1,2,4-triazine), 40.57 - 40.46 (-CH-).

Tri[6-(5'-fluoro-2'-triphenxylphosphin-iminophenyl)-5-oxo-1,2,-4-triazine-3'yl]trimercaptoacetic acid (8)

A mixture of $2(0.03 \mathrm{~mol})$ and 1,1,1-trichloroacetic acid $(0.01 \mathrm{~mol})$ in DMF $(20 \mathrm{ml})$ warm for $30 \mathrm{~min}$ then cold and poured on to ice. The produced solid filtered off and crystallized from Et OH to give $\mathbf{8}$ as reddish crystals. Yield (60\%); m.p. $189^{\circ} \mathrm{C}-190^{\circ} \mathrm{C}$. Analytical data: Found $\mathrm{C}=63.89, \mathrm{H}=3.45, \mathrm{~F}=3.55 \mathrm{~N}, 10.67, \mathrm{~S}=$ 5.83\%. Calculated for $\mathrm{C}_{83} \mathrm{H}_{58} \mathrm{~F}_{3} \mathrm{~N}_{12} \mathrm{O}_{5} \mathrm{P}_{3} \mathrm{~S}_{3}(1548) ; \mathrm{C}=64.34, \mathrm{H}=3.74, \mathrm{~F}=3.68, \mathrm{~N}=10.85, \mathrm{~S}=6.2 \%$. UV $\left(\lambda_{\max }\right.$ EtOH) $359 \mathrm{~nm}$. IR $v \mathrm{~cm}^{-1}=3500-3100(\mathrm{~b}, 3 \mathrm{NH}, \mathrm{OH}) 1716(\mathrm{C}=\mathrm{O}), 1624(\mathrm{NH}=\mathrm{OH}$ of 1,2,4-triazinone) 1537 $(\mathrm{C}=\mathrm{N}) 1471$ (aliphatic CH). $1390(\mathrm{P}=\mathrm{N}), 1300$ (NCSN), 1260 (C-F), 1200 (C-S), 1645 (P-N), 920, 850, 780 (aryl CH), $650(\mathrm{C}-\mathrm{F}) .{ }^{1} \mathrm{H}$ NMR $(\mathrm{DMSO})=\delta 12.95,12.72,12.33($ each s, 3H, NH), $10.84(\mathrm{~s}, 1 \mathrm{H}, \mathrm{OH}), 8.51,8.23$, 8.01, 7.92, 7.89, 7.71, 7.7, 7.69, 7.65, 7.63, 7.59, 7.58, 7.57 - 7.54, 7.50 - 7.47, 7.40 - 7.37, 7.33 - 7.31, 7.02 6.98, $6.86-6.83$ (aromatic CH). ${ }^{13} \mathrm{C}$ NMR = (DMSO) $\delta 179.61(\mathrm{C}=\mathrm{S}), 163(\mathrm{C}=\mathrm{O}), 159.5(\mathrm{C}-\mathrm{S}), 157(\mathrm{C}-\mathrm{F})$ 138.58 $(\mathrm{C}=\mathrm{N}), 137.79(\mathrm{C}=\mathrm{N}), 132.91(\mathrm{C}=\mathrm{N}), 131.99-107.93$ (aromatic $\mathrm{CH}), 77.92,77.49\left(\mathrm{C}_{5}-\mathrm{C}_{6}\right.$ of 1,2,4-triazine).

6(5'-Fluoro-2'-triphenylphosphin-iminophenyl)2,-4-dihydro-thiazolo[3,2-b][1,2,4]triazine-3,7-dione (9)

Equimolar mixture of 2 and monochloroactic acid in DMF $(20 \mathrm{ml})$ reflux for $2 \mathrm{~h}$ then cold and poured onto ice. The solid obtained filtered off and crystallized from dioxan to give 9 as brown ppt, Yield (60\%) m.p. $224^{\circ} \mathrm{C}$. Compound $6(0.50 \mathrm{mg})$ heat above its melting point $\left(60^{\circ} \mathrm{C}\right.$ higher) for $10 \mathrm{~min}$, cold then treat with $\mathrm{MeOH}$. The solid produced filtered off and crystallized from dioxan to give 9 as brown ppt. Yield (58\%), m.p. $225^{\circ} \mathrm{C}-227^{\circ} \mathrm{C}$. Analytical data Found $\mathrm{C}=64.40, \mathrm{H}=3.51, \mathrm{~F}=3.35, \mathrm{~N}=9.93, \mathrm{~S}=5.48 \%$ Calculated for $\mathrm{C}_{29} \mathrm{H}_{20} \mathrm{FN}_{4} \mathrm{O}_{2} \mathrm{PS}(538)$; $\mathrm{C}=64.68, \mathrm{H}=3.71 \mathrm{~F}=3.53, \mathrm{~N}=10.40 . \mathrm{S}=5.94 \% . \mathrm{UV}:\left(\lambda_{\max } \mathrm{EtOH}\right) 352 \mathrm{~nm} . \mathrm{IR} v \mathrm{~cm}^{-1}=3204(\mathrm{~b}-\mathrm{OH}), 1694$ $(\mathrm{C}=\mathrm{O}), 1623(\mathrm{C}=\mathrm{N}), 15,636,1475\left(\mathrm{CH}_{2}\right), 1380(\mathrm{P}=\mathrm{N})$, 12,999 (C-F), 1148 (C-S) 816, (aromatic CH), 711 (C-F). ${ }^{1} \mathrm{H}$ NMR $(\mathrm{DMSO})=\delta 10.79$ (s, $1 \mathrm{H}$, Phenolic $\left.\mathrm{OH}\right), 8.23$ (s, $1 \mathrm{H}, \mathrm{CH}$ of thazole), $7.95-7.47,7.35,7.35,6.98$, 6.80 (aromatic CH). ${ }^{13} \mathrm{C}$ NMR = (DMSO): $\delta 167.21(\mathrm{C}=\mathrm{S}), 147.47,(\mathrm{C}=\mathrm{O}), 136.64(\mathrm{C}-\mathrm{F}), 132.97(\mathrm{C}=\mathrm{N}), 131.92$ 128.54, 118.80 - 118.14, 113.79 - 113.73 (aromatic carbons), $111.04-110.99(-\mathrm{CH}=), 77.80,77.38\left(\mathrm{C}_{5}-\mathrm{C}_{6}\right.$ of 1,2,4-triazine).

6(5'-Fluoro-2'-triphenylphosphiniminophenyl)-5-oxo-3-(cyanomethylthia)-2H-1,2,4-triazine (10)

A mixture of $2(0.01 \mathrm{~mol})$ and chloroacetinitrile $(0.01 \mathrm{~mol})$ in DMF $(20 \mathrm{ml})$ warm $(10 \mathrm{~min})$ then cold and poured onto ice. The result solid filtered off and crystallized from dioxan to give $\mathbf{1 0}$ as faint Yellow crystals. Yield (70\%); m.p. $214^{\circ} \mathrm{C}-215^{\circ} \mathrm{C}$. Analytical data: Found $\mathrm{C}=64.39, \mathrm{H}=3.58, \mathrm{~F}=3.11, \mathrm{~N}, 12.85, \mathrm{~S}=5.75 \%$. Calculated for $\mathrm{C}_{29} \mathrm{H}_{21} \mathrm{FN}_{5} \mathrm{OPS}$ (537); $\mathrm{C}=64.80, \mathrm{H}=3.91, \mathrm{~F}=3.53, \mathrm{~N}=13.03, \mathrm{~S}=5.95 \% . \mathrm{M} / \mathrm{Z}=537$ (5\%) 281 (20), 207 (60), 149 (20), 113 (30), 85 (100), 58 (100). UV: ( $\left.\lambda_{\max } \mathrm{EtOH}\right) 321 \mathrm{~nm}$. IR vcm ${ }^{-1}=3424,3167$ (NH, $\mathrm{S}-\mathrm{CH}=\mathrm{C}=\mathrm{NH}) 2100-2085(\mathrm{C} \equiv \mathrm{N}), 1646(\mathrm{C}=\mathrm{O}), 1595(\mathrm{C}=\mathrm{N}), 1481\left(\mathrm{CH}_{2}\right), 1370(\mathrm{P}-\mathrm{N})$, 967, 839, 762 (aryl $\left.\mathrm{CH}\right)$, $700(\mathrm{C}-\mathrm{F}) .{ }^{1} \mathrm{H}$ NMR $(\mathrm{DMSO})=\delta 13.90,(\mathrm{~s}, 1 \mathrm{H}, \mathrm{NH}), 12.76(\mathrm{~s}, 1 \mathrm{H}, \mathrm{HC}=\mathrm{NH}), 8.22-6.81($ aromatic $\mathrm{CH}), 4.69$ $(1 \mathrm{H}, \mathrm{HC}=\mathrm{NH}) 2.59\left(2 \mathrm{H}, \mathrm{CH}_{2}\right) \cdot{ }^{13} \mathrm{C}$ NMR (DMSO): $\delta 158.11(\mathrm{C}=\mathrm{O}), 147.0(\mathrm{C}-\mathrm{F}) 132(\mathrm{C}=\mathrm{N}), 131.86-128.44$ (aromatic carbons), $112.21(\mathrm{C} \equiv \mathrm{N}), 77.96,77.53\left(\mathrm{C}_{5}-\mathrm{C}_{6}\right.$ of 1,2,4-triazine), $40.133(-\mathrm{CH}=\mathrm{NH}), 33.63\left(\mathrm{CH}_{2}\right)$.

\section{3-Amino-6(5'-fluoro-2'-triphenylphosphiniminophenyl)-thiazolo[3,2-b][1,2,4]triazine-7-one (11)}

Compound $10(0.5 \mathrm{gm})$ in DMF $(20 \mathrm{ml})$ warm for $2 \mathrm{~h}$ then cooled and poured onto ice. The solid produced filtered off and crystallized from EtOH to give $\mathbf{1 1}$ as broom ppt, Yield (66\%); m.p. $223^{\circ} \mathrm{C}-225^{\circ} \mathrm{C}$. Analytical data: Found $\mathrm{C}=64.51 \mathrm{H}=3.38, \mathrm{~F}=3.21 \mathrm{~N}=12.55, \mathrm{~S}=5.62 \%$. Calculated for $\mathrm{C}_{29} \mathrm{H}_{21} \mathrm{FN}_{5} \mathrm{OPS}$ (537), $\mathrm{C}=64.80$, $\mathrm{H}=3.91, \mathrm{~F}=3.53, \mathrm{~N}=13.03, \mathrm{~S}=5.95 \% . \mathrm{M} / \mathrm{Z}, 537$ (2\%), 370 (2), 226 (2), 168 (100), 140 (60), 114 (30), 62 (18), 70 (18). IR vcm ${ }^{-1}=3348,\left(\mathrm{~b}-\mathrm{NH}_{2}\right), 16430(\mathrm{C}=\mathrm{O}), 1383(\mathrm{P}=\mathrm{N}), 1250(\mathrm{C}-\mathrm{F}), 1086$ (C-S), 1045 (P-N), 878 $\left(\right.$ aryl CH). ${ }^{1} \mathrm{H}$ NMR $(\mathrm{DMSO})=\delta 8.11(\mathrm{~s}, 1 \mathrm{H},=\mathrm{CH}$ thiaszole $), 7.72-7.011,6.98-6.80$ (aromatic $\left.\mathrm{CH}\right), 3.99-$ $3.84\left(2 \mathrm{H}-\mathrm{NH}_{2}\right) .{ }^{13} \mathrm{C}$ NMR $(\mathrm{DMSO})=162.54(\mathrm{C}=\mathrm{O}), 132.16(\mathrm{C}-\mathrm{F}), 132.00(\mathrm{C}=\mathrm{N}), 131.99(\mathrm{C}-\mathrm{S}), 131.66-$ 131.64 (=CH-), 128.61 - 120.55 (aromatic carbons), 77.59, 77.38 ( $\mathrm{C}_{5}-\mathrm{C}_{6}$ of 1,2,4-triazine), 40.51 (-N-C=N).

3-(4'-Fluoro benzoyl)amino-6-(5'-fluoro-2'-triphenylphosphiniminophenyl)-thiazolo[3,2-b][1,2,4] triazine-7-one (12)

Equimolar mixture of $\mathbf{1 1}$ and 4-fluorobenzoyl chloride in DMF (20 ml) warm for $10 \mathrm{~min}$ then cold and poured onto ice. The resulted solid filtered off and crystallized from EtOH to give $\mathbf{1 2}$ as deep-Yellowish crystals. Yield (75\%). m.p. $205^{\circ} \mathrm{C}-207^{\circ} \mathrm{C}$. Analytical data: Found $\mathrm{C}=65.19 \mathrm{H}=3.41, \mathrm{~F}=5.49 \mathrm{~N}=10.51, \mathrm{~S}=4.59 \%$. Calculated for $\mathrm{C}_{36} \mathrm{H}_{25} \mathrm{~F}_{2} \mathrm{~N}_{5} \mathrm{O}_{2} \mathrm{PS}(660), \mathrm{C}=65.55, \mathrm{H}=3.80, \mathrm{~F}=5.76, \mathrm{~N}=10.60, \mathrm{~S}=4.80$. IR $v \mathrm{~cm}^{-1}=3342$, (b-NH), 1651 (b, 2C=O), $1381(\mathrm{P}=\mathrm{N}), 1326$ (NCSN) 1230 (C-F), 1086 (C-S), 1045 (P-N), 879 (aryl CH). ${ }^{1} \mathrm{H}$ NMR $(\mathrm{DMSO})=\delta 13.70(\mathrm{~s}, 1 \mathrm{H}, \mathrm{NH}), 9.89(\mathrm{~s},=\mathrm{CH}$ of thiazole) 8.411, 8.17, $8.07-8.05,8.01,7.99,7.95,7.79,7.66-$ 
7.64, 7.44 - 7.42, 7.28, 7.27 (aromatic CH). ${ }^{13} \mathrm{C}$ NMR = (DMSO): $\delta 167.53$ (C=O), 162.54 (C=O) 138.59 (C-F) $132.25(\mathrm{C}-\mathrm{N}), 132.19(\mathrm{C}=\mathrm{N}), 129.33$ - 127.27, 117.78 - 115.17, 112.15, 12.09, 110.52, 108.12, 107.95 (aromatic carbons), 77.64. $77.43\left(\mathrm{C}_{5}-\mathrm{C}_{6}\right.$ of 1,2,4-triazine).

\section{Schiff base (13)}

Equimolar amounts of $\mathbf{1 1}$ and 4-fluorobenzaldehyde in absolute ethanol $(20 \mathrm{ml})$ reflux for $30 \mathrm{~min}$ then cooled. The solid thus obtain filtered off and crystallized from EtOH to give $\mathbf{1 3}$ as Yellowish ppt. Yield (70\%); m.p. $248^{\circ} \mathrm{C}-250^{\circ} \mathrm{C}$. Analytical data: Found $\mathrm{C}=66.85, \mathrm{H}=3.61, \mathrm{~F}=5.75 \mathrm{~N}=10.59, \mathrm{~S}=4.71 \%$. Calculated for $\mathrm{C}_{36} \mathrm{H}_{24} \mathrm{~F}_{2} \mathrm{~N}_{5} \mathrm{OPS}$ (643), $\mathrm{C}=67.18, \mathrm{H}=3.73, \mathrm{~F}=5.90, \mathrm{~N}=10.88, \mathrm{~S}=4.97 \% . \mathrm{IR} v \mathrm{~cm}^{-1}=3100,2880($ aromatic \& aliphatic CH), 1700 (C=O), 1600 (C=N), 1483 (C-P), 1370 (P=N), 1230 (C-F), 1200 (C-S), 1045 (P-N), 880, 840, 810 (aryl CH), $650(\mathrm{C}-\mathrm{F}){ }^{1} \mathrm{H}$ NMR (DMSO) $=\delta 9.97(\mathrm{~s}, 1 \mathrm{H},-\mathrm{CH}=\mathrm{N}-), 8.62(\mathrm{~s}, 1 \mathrm{H},-\mathrm{CH}=$ thiazole) 8.23, 8.22, 8.09 - 8.00, 7.94 - 7.92, 7.71 - 7.63, 7.56 - 7.53, 7.49 - 7.45, 7.26 - 7.23, 7.12 - 7.10, 7.0 - 6.96, 6.89 - 6.84, 6.81 - 6.79 (aromatic $\mathrm{CH}$ ).

6-(5'-Fluoro-2'-triphenyl phosphiniminophenyl)-3-oxo-3phenyl-thiazolo[3,2-b][1,2,4]triazine-7-one (14)

A mixture of $2(0.01 \mathrm{~mol})$ and phenacylbromide $(0.01 \mathrm{~mol})$ in ethanolic $\mathrm{KOH},(20 \mathrm{ml}, 5 \%)$ reflux for $2 \mathrm{~h}$, cold then poured onto ice-HCl. The solid produced filtered off and crystallize from dioxan to give $\mathbf{1 4}$ as brown ppt. Yield (60\%); m.p. $>300^{\circ} \mathrm{C}$. Analytical data: Found $\mathrm{C}=69.88, \mathrm{H}=3.59, \mathrm{~F}=3.01 \mathrm{~N}, 9.00, \mathrm{~S}=5.13 \%$, Calculated for $\mathrm{C}_{35} \mathrm{H}_{24} \mathrm{FN}_{4} \mathrm{OPS}(598) ; \mathrm{C}=70.23, \mathrm{H}=4.01, \mathrm{~F}=3.17, \mathrm{~N}=9.36 \mathrm{~S}=5.35 \%$. IR v cm${ }^{-1}=3080,3030$ (aromatic CH), $1680(\mathrm{C}=\mathrm{O}), 1380(\mathrm{P}=\mathrm{N}), 1240(\mathrm{C}-\mathrm{F}), 1180(\mathrm{C}-\mathrm{S}), 1045(\mathrm{P}-\mathrm{N}), 880,850$ (aryl CH).

\section{Diaylthioether (16)}

A mixture of $2(0.01 \mathrm{~mol})$ and Schiff base $15(0.01 \mathrm{~mol})$ in dry $\mathrm{C}_{6} \mathrm{H}_{6}(100 / \mathrm{ml})$ reflux $8 \mathrm{~h}$, cold and used petereither $100^{\circ} \mathrm{C}-120^{\circ} \mathrm{C}$ to complete precipitation. The solid obtained filtered off and crystallized dioxan to give $\mathbf{1 6}$ as Yellowish crystals. Yield (80\%); m.p. $204^{\circ} \mathrm{C}-205^{\circ} \mathrm{C}$. Analytical data: Found $\mathrm{C}=66.53, \mathrm{H}=4.31, \mathrm{~F}=4.44$, $\mathrm{N}=11.88, \mathrm{~S}=3.66 \%$, Calculated for $\mathrm{C}_{45} \mathrm{H}_{36} \mathrm{~F}_{2} \mathrm{~N}_{7} \mathrm{O}_{2} \mathrm{PS}(807) ; \mathrm{C}=66.91, \mathrm{H}=4.46, \mathrm{~F}=4.70, \mathrm{~N}=12.14, \mathrm{~S}=$ 3.96\%. M/Z = (807.0.0), 580 (5), 515 (4), 462 (8), 423 (10), 370 (5), 339 (20), 282 (20), 225 (20), 207 (60), 176 (100), 149 (56), 119 (38), 85 (90), 58 (100). UV: ( $\lambda_{\max }$ EtOH) $323 \mathrm{~nm} . \mathrm{IR} v \mathrm{~cm}^{-1}=3332(\mathrm{NH}), 2973,2886$ (aliphatic CH), $1636(\mathrm{C}=\mathrm{O}), 1488\left(\mathrm{CH}_{3}\right), 1381(\mathrm{P}=\mathrm{N}), 1324$ (NCSN), 1250 (C-F), 1086 (C-S), 1045, (P-N), 880, 755 (aryl CH). ${ }^{1} \mathrm{H}$ NMR $(\mathrm{DMSO})=\delta$ 12.77, $(\mathrm{s}, 1 \mathrm{H}, \mathrm{NH}), 10.75(\mathrm{~s}, 1 \mathrm{H}, \mathrm{NH}), 9.68(\mathrm{~s}, 1 \mathrm{H}, \mathrm{S}-\mathrm{CH}-\mathrm{Ar}), 8.23,7.83-$ 7.28, 7.27 - 7.00, 6.99 - 6.84 (aromatic $\mathrm{CH}), 3.69\left(\mathrm{~s}, \mathrm{CH}_{3}-\mathrm{N}\right) 2.79\left(\mathrm{~s}, \mathrm{CH}_{3}-\mathrm{C}\right) .{ }^{13} \mathrm{C}$ NMR (DMSO): $\delta 164.73(\mathrm{C}=$ O), 163.08 (C=O), 160.63, 155.18 (C-F), 151.9 (C-S), 134.64, 134.25, 134.23 (C=N), 129.487 - 115.492 (aromatic carbons), 77.72, 77.50 ( $\mathrm{C}_{5}-\mathrm{C}_{6}$ of 1,2,4-triazine), $67.00(\mathrm{~S}-\mathrm{CH}=\mathrm{NH}), 39.95-39.81,39.67-39.55\left(2 \mathrm{CH}_{3}\right)$.

2,3-Diaryl-2,3-dihydro-4-thioxo-7-(5'-fluoro-2'-triphenylphosphiniminophenyl)-1,3,5-thiazolo[3,2-b] $[1,2,4]$ triazine-8-one $(17)$

A mixture of $16(0.01 \mathrm{~mol})$ and $\mathrm{CS}_{2}(5 \mathrm{ml})$ in DMF $(20 \mathrm{ml})$ reflux for $4 \mathrm{~h}$, cold then powered onto ice. The resultant solid filtered off and crystallized from dioxan to give $\mathbf{1 7}$ as yellowish crystals. Yield (75\%), m.p. $254^{\circ} \mathrm{C}$ $255^{\circ} \mathrm{C}$. Analytical data: Found $\mathrm{C}=64.88, \mathrm{H}=3.85, \mathrm{~F}=4.38, \mathrm{~N}=11.40, \mathrm{~S}=7.45 \%$, Calculated for $\mathrm{C}_{46} \mathrm{H}_{34} \mathrm{~F}_{2} \mathrm{~N}_{7} \mathrm{O}_{2} \mathrm{PS}_{2}$ (849); $\mathrm{C}=65.01, \mathrm{H}=4.00, \mathrm{~F}=4.47, \mathrm{~N}=11.54, \mathrm{~S}=7.53 \% ; \mathrm{M} / \mathrm{Z}$ (849, 0.0\%), 370 (2), 329 (40), 290 (100), 159 (100), 128 (100), 102 (100), 96 (100), 65 (100). UV: ( $\lambda_{\max }$ EtOH) $34.7 \mathrm{~nm}$. IR vcm ${ }^{-1}=2873$ (aliphatic CH), $1684(\mathrm{C}=\mathrm{O}), 1614.1593(\mathrm{C}=\mathrm{N})$ 1475, $1425\left(\mathrm{CH}_{3}\right), 1318(\mathrm{P}=\mathrm{N}), 1264(\mathrm{C}-\mathrm{F}), 1199(\mathrm{C}=\mathrm{S}), 1130$ (C-S), $1052(\mathrm{P}-\mathrm{N}), 985,899,854,814,732(\operatorname{aryl} \mathrm{CH}) .{ }^{13} \mathrm{C}$ NMR $(\mathrm{DMSO})=\delta 179.70(\mathrm{C}=\mathrm{S}), 163.07(\mathrm{C}=\mathrm{O})$, 155.08 (C-F), 138.62 (C=N, 1,2,4-triazine), 132.23, 132.13 (C=C pyrazole), 129.43 - 115.44, 112.09 - 107.96 (aromatic carbons), 77.71, 77.28 ( $\mathrm{C}_{5}-\mathrm{C}_{6}$ of 1,2,4-triazine) 66.94 (S-CH-NH), 40.57, $39.76\left(2 \mathrm{CH}_{3}\right)$.

\section{Di-Heteroaryldisulfide (18)}

Compound $2(0.05 \mathrm{gm})$ and $\mathrm{FeCl}_{3}(0.5 \mathrm{gm})$ in $\mathrm{MeOH}(20 \mathrm{ml})$ reflux for $3 \mathrm{~h}$, then filtered. The solid produced filtered off and crystallized from dioxan to give 18 as deep-yellowish crystals. Yield (80\%), m.p. $238^{\circ} \mathrm{C}-240^{\circ} \mathrm{C}$. Analytical data: Found $\mathrm{C}=64.85, \mathrm{H}=10.89, \mathrm{~F}=3.55, \mathrm{~N}=10.89, \mathrm{~S}=6.22 \%$. Calculated for $\mathrm{C}_{54} \mathrm{H}_{38} \mathrm{~F}_{2} \mathrm{~N}_{8} \mathrm{O}_{2} \mathrm{P}_{2} \mathrm{~S}_{2}$ (994); $\mathrm{C}=65.19, \mathrm{H}=11.26, \mathrm{~F}=3.82, \mathrm{~N}=11.26, \mathrm{~S}=6.43 \%$. IR $v \mathrm{~cm}^{-1}=3300,3200(\mathrm{NH}, \mathrm{NH}), 1680(\mathrm{C}=\mathrm{O})$, $1600(\mathrm{C}=\mathrm{N}), 1350(\mathrm{P}=\mathrm{N}), 1100(\mathrm{C}-\mathrm{S}), 1040(\mathrm{P}-\mathrm{N}), 900,850,800(\operatorname{aryl} \mathrm{CH}), 650(\mathrm{C}-\mathrm{F}) .{ }^{1} \mathrm{H}$ NMR $(\mathrm{DMSO})=\delta$ 14.55, 12.78 (each s, 2H, NH, NH), 8.20 - 6.85 (aromatic CH). ${ }^{13} \mathrm{C}$ CNMR (DMSO): $\delta$ 179.90, 179.74 (2C-S), 159 - 66, $158.66(2 \mathrm{C}=\mathrm{O}), 138.63(\mathrm{C}-\mathrm{F}), 132.18(\mathrm{C}=\mathrm{N}), 121.12$ - 107.95 (aromatic carbons), 77.65, $77.43\left(\mathrm{C}_{5}-\mathrm{C}_{6}\right.$ of 1,2,4-triazine).

6-(5'-Fluoro-2'-triphenylphosphiniminophenyl)-5-oxo-2H-1,2,4-triazine-3-sulfonic acid (19)

Compound $2(0.05 \mathrm{gm})$ in ethanol $(10 \mathrm{ml})$ and $\mathrm{H}_{2} \mathrm{O}_{2},(0.5 \mathrm{ml})$ add with stirring for $2 \mathrm{~h}$. The solid obtained filtered off and crystallized from EtOH to give 19 as yellowish crystals yield $(75 \%)$; m.p. $258^{\circ} \mathrm{C}-260^{\circ} \mathrm{C}$. Analyti- 
cal data: Found $\mathrm{C}=59.00, \mathrm{H}=3.44, \mathrm{~F}=3.25, \mathrm{~N}=9.87, \mathrm{~S}=5.45 \%$. Calculated for $\mathrm{C}_{27} \mathrm{H}_{20} \mathrm{FN}_{4} \mathrm{O}_{4} \mathrm{PS}$ (546), $\mathrm{C}=$ 59.34, $\mathrm{H}=3.66, \mathrm{~F}=3.47, \mathrm{~N}=10.25, \mathrm{~S}=5.86 \%$. IR $v \mathrm{~cm}^{-1}=3300(\mathrm{NH}), 1696(\mathrm{C}=\mathrm{O}), 1390(\mathrm{NCSN}), 1360$ $(\mathrm{P}=\mathrm{N}), 879,820,780$, (aryl CH). $670(\mathrm{C}-\mathrm{F}) .{ }^{1} \mathrm{H}$ NMR (DMSO) $=\delta 12.79(\mathrm{~s}, 1 \mathrm{H}, \mathrm{NH}), 10.72\left(\mathrm{~s}, 1 \mathrm{H}, \mathrm{SO}_{2}-\mathrm{OH}\right)$, 8.13 - 6.76 (aromatic CH). ${ }^{13} \mathrm{C}$ NMR (DMSO): $\delta 179.75(-\mathrm{S}=\mathrm{O}), 163.09(\mathrm{C}=\mathrm{O}), 159.67(\mathrm{C}-\mathrm{F}), 138.62(\mathrm{C}=\mathrm{N})$, 132.23 (C-S), 121.08 - 107.95 (aromatic carbons), 77.60, 77.39 ( $\mathrm{C}_{5}-\mathrm{C}_{6}$ of 1,2,4-triazine).

\section{6-(5'-Fluoro-2'-triphenylphosphiniminophenyl)-1,2,4-triazine-3,5-(2H, 4H)dione (20)}

Compound $2(0.05 \mathrm{gm})$ in ethanol $(10 \mathrm{ml})$ and $\mathrm{KMnO}_{4}$ solution (ethanolic $\left.1 \%, 1 \mathrm{ml}\right)$ add drop wise then stirring for $2 \mathrm{~h}$. The produced solid filtered off and crystallized from Et $\mathrm{OH}$ to give $\mathbf{2 0}$ as yellowish crystals yield (50\%); m.p. $273^{\circ} \mathrm{C}-275^{\circ} \mathrm{C}$. Analytical data: Found $\mathrm{C}=66.89, \mathrm{H}=4.01, \mathrm{~N}=11.35, \mathrm{~F}=3.55$. Calculated for $\mathrm{C}_{27} \mathrm{H}_{20} \mathrm{FN}_{4} \mathrm{O}_{2} \mathrm{P}$ (482), $\mathrm{C}=67.21, \mathrm{H}=4.14, \mathrm{~N}=11.61, \mathrm{~F}=3.94 . \mathrm{M} / \mathrm{Z}:$ (482, 0.0\%), 370 (10), 206 (101, 148, (16), 128 (24), 110 (35), 96 (55), 83 (78), 68 (100). IR $v \mathrm{~cm}^{-1}=3426,3259,3170(\mathrm{OH}, \mathrm{NH}, \mathrm{NH}), 1766,1681(2 \mathrm{C}=\mathrm{O})$, $1619(\mathrm{C}=\mathrm{N}) 1452(\mathrm{C}-\mathrm{P}) 1301(\mathrm{P}=\mathrm{N})$, 1252, (C-F) $1048(\mathrm{P}-\mathrm{N}), 905,861,820,802,784,761(\mathrm{CH}), 687(\mathrm{C}-\mathrm{F}),{ }^{1} \mathrm{H}$ NMR $($ DMSO $)=\delta 12.73,10.82($ each s, NH, OH), $7.88-6.84$ (aromatic $\mathrm{CH}) .{ }^{13} \mathrm{C}$ NMR $=\delta 153.58(\mathrm{C}=\mathrm{O})$, $152.78(\mathrm{C}=\mathrm{O}), 147.84(\mathrm{C}-\mathrm{F}) .132 .58(\mathrm{C}=\mathrm{N}) 130.51$ - 111. (Aromatic carbons), 77.85, $77.60\left(\mathrm{C}_{5}-\mathrm{C}_{6}\right.$ of 1,2,4-triazine).

\section{Results and Discussion}

\subsection{Chemistry}

A recent work on the synthesis and chemistry of bioactive sulfur bearing 1,2,4-triazinone moiety was reported [16] [19]. In continuation of this attitude the present investigation reports the synthesis of fluorine and phosphorus-substituted 6-amino-phenyl-3-thioxo-1,2,4-triazin-5-(2H, 4H) one (1) and study that behavior towards various alkylating agents. Treatment of 5-fluoroisatin with thiosemicarbozide in alkaline medium [14] [15] produced 6-(2'-amino-5'-fluorophenyl-3-thioxo-1,2,4-triazin-5-(2H'4H) one (1). Warm compound 1 with triphenylphosphine in acetonitrile produced the yield $\mathbf{2}$ (Scheme 1 ).

In the imino [yield, 2] a negative charge of nitrogen is bonded to positive charge of phosphorus stabilized by partial overlap of the filled N-P orbital. This stabilization increase due to the charge on the $\alpha$-carbon atom is spread by 1,2,4-triazine resonance. Abdel-Rahman [14] [15] reported that N-alkyl of 3-thioxo-1,2,4-triazinones exhibited a wide biological spectrum anti HIV and anticancer properties. Similarly, hydroxyl methylation of compound 2 by boil with formaldehyde-methanolproduced 2,4-di(hydroxylmethyl)-6-(5'fluoro-2'-triphenylphosphiniminophenyl)-3-thioxo-1,2,4-triazin-5-one (3). Also, reflux of compound 2 with secondary and primary amines such as piper dine, 4-fluoroaniline and 4-amino-antipyrine in the presence of formaldehyde methanol, furnished the Mannich bases 4 and 5 (Scheme 2).

Formation of $\mathbf{3}$ and $\mathbf{4}$ was may be as (Figure $\mathbf{1}$ ).

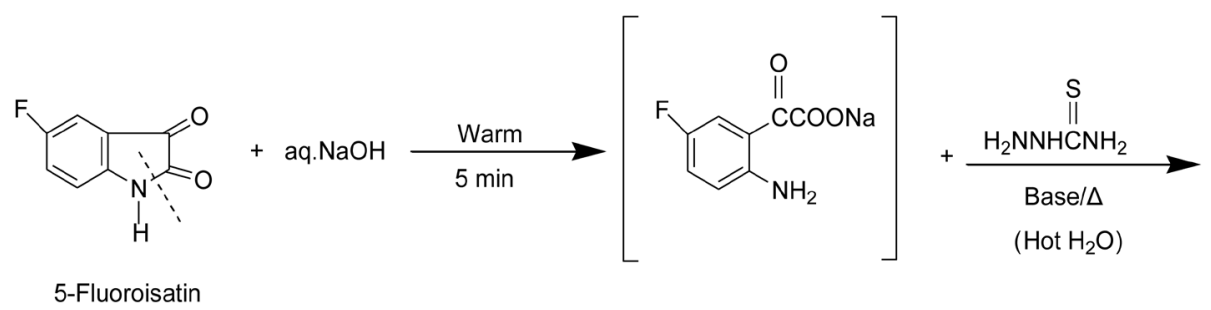

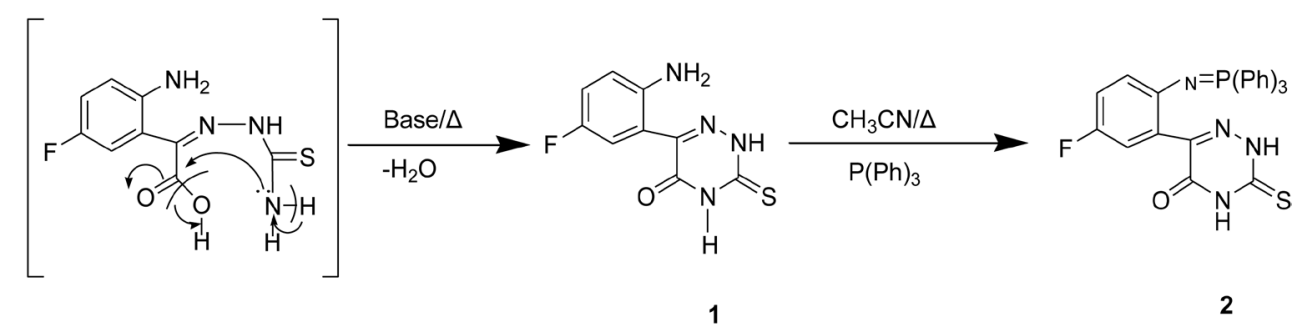




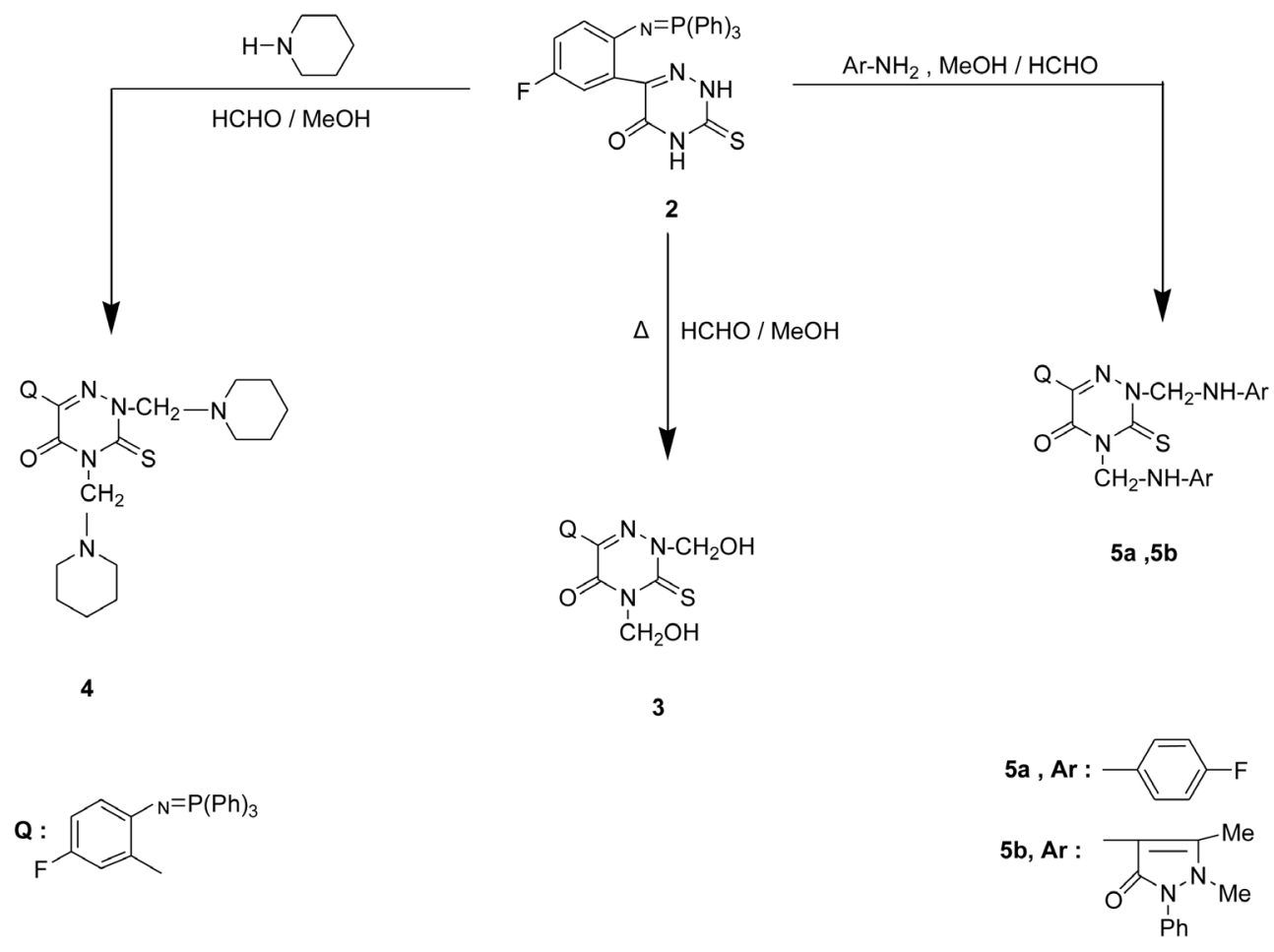

Scheme 2. Formation of compounds 3 - 5.
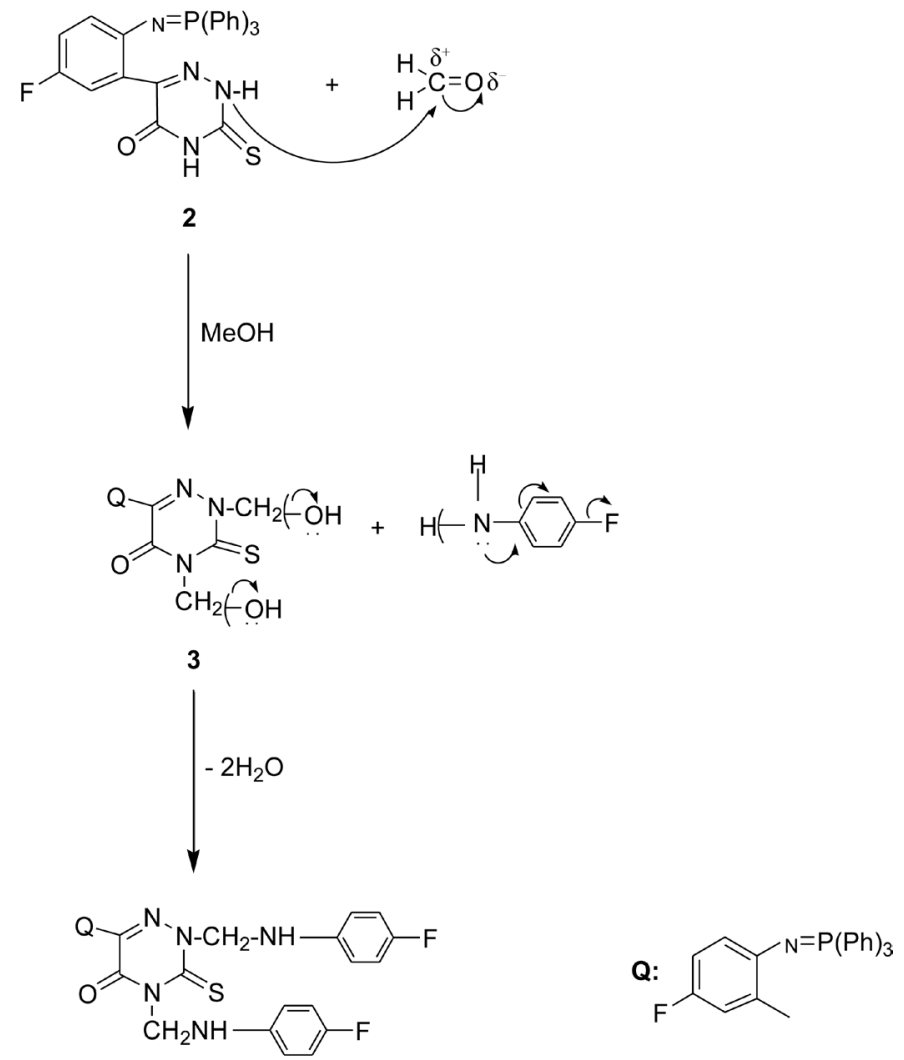

$5 a$

Figure 1. Formation of compounds $3 \& 5$ a. 
Due to a higher nucleophilicity of sulfur atoms, the direct displacement of an acidic proton of mercapto group by a simple electrophile can be easily occur via treatment of compound $\mathbf{2}$ with haloacetic acids. Thus treatment of compound 2 with halo aliphatic acids such as mono/di/trichloroacetic acids in DMF afforded the substituted thiaacetic acids 6-8 (Scheme 3).

The multicomponent reaction (MCR) was considered as powerful synthetic tool for preparing target molecules of biological relevance in an efficient manner. Thus, treatment of compound $\mathbf{2}$ with active methylene reagents as chloroacetonitrile in warm DMF [20] produced 3-cyanomethyl thai-6-iminophosphorane-1,2,4-trinazin5-(2H)one (10). The latter compound $\mathbf{1 0}$ use for the synthesis of thiazolo [3,2-b][1,2,4]triazinones (11-13) systems (Scheme 4). Acidic hydrolysis of $\mathbf{1 0}$ by warm with diluted $\mathrm{HCl}$ for short time (10 min) yielded the compound 6. Boil compound 6 with DMF along time afforded 6-iminophosphorane-2,3-dihydoro-thiazolo [3,2-b] $[1,2,4]$ triazine-3,7-dione (9) (Scheme 4).

Heat compound $\mathbf{1 0}$ on heating with DMF a long time (2 hours), produced 3-aminothiazolo-1,2,4-triazine $\mathbf{1 1}$. Presence of an amino group in structure $\mathbf{1 1}$ was deduced from treat with 4-fluorobenzoylchloride (DMF) and/or with 4-fluorobenzaldehyde (EtOH) yield the anilido 12 and/or Schiff's base 13 (Scheme 4). Treatment of compound 2 with $\alpha$, $\beta$-bifunctional oxygen-halogen reagents as phenacyl bromide in ethanolic $\mathrm{KOH}$, yielded 3phenyl-6-iminophosphorane-thiazolo [3,2-b][1,2,4]triazin-7-one (14) (Scheme 5). The nitrogen-sulfur containing fused heterobicyclic structures have demonstrated a high degree of binding affinity when they serve as Ligands for various biological receptors [12] [13]. Thus addition of Mercator group (as nucleophilic) of compound $\mathbf{2}$ to an Schiff's base $\mathbf{1 5}$ in boil dry dioxan yielded the thioether16, which upon ring closure reaction by reflux with $\mathrm{CS}_{2}$ in DMF furnished 2,3-diaryl-2,3-dihydro-7-iminophosphorane-4-thioxo-1,3,5-thiadiazino[3,2-b] [1,2,4]-triazin-8-one (17) (Scheme 5).

Abdel-Rahman et al. [21]-[25] reported that thioethers, sulfide and sulfonic acid bearing a 1,2,4-triazine moieties. Exhibited a very interesting medicinal activity as anti-HIV and anticancer agents. Recently, Slawinski et al. [25] synthesized 2-mercaptobenzene sulfonamide bearing a 1,2,4-trinzines exhibited a significant activity against cell lines of colon cancer, renal cancer, and melanoma, as well as good selectivity toward non-small cell lung cancer. Similarly, oxidation of compound 2 via treatment with $\mathrm{FeCl}_{3}$ in boiling methanol and/or with $\mathrm{H}_{2} \mathrm{O}_{2}$ in ethanol by stirred at room temperature furnished the disulfide $\mathbf{1 8}$ and/or 3-sulfonic-1,2,4-triazinone 19. Finally, treatment of $\mathbf{2}$ with ethanolic $\mathrm{KMnO}_{4}$ at room temperature [21] led to the direct formation of 6-(5'-fluoro2-triphenylphosphiniminophenyl)-1,2,4-triazin-3,5(2H, 4H)dione (20) (Scheme 5).
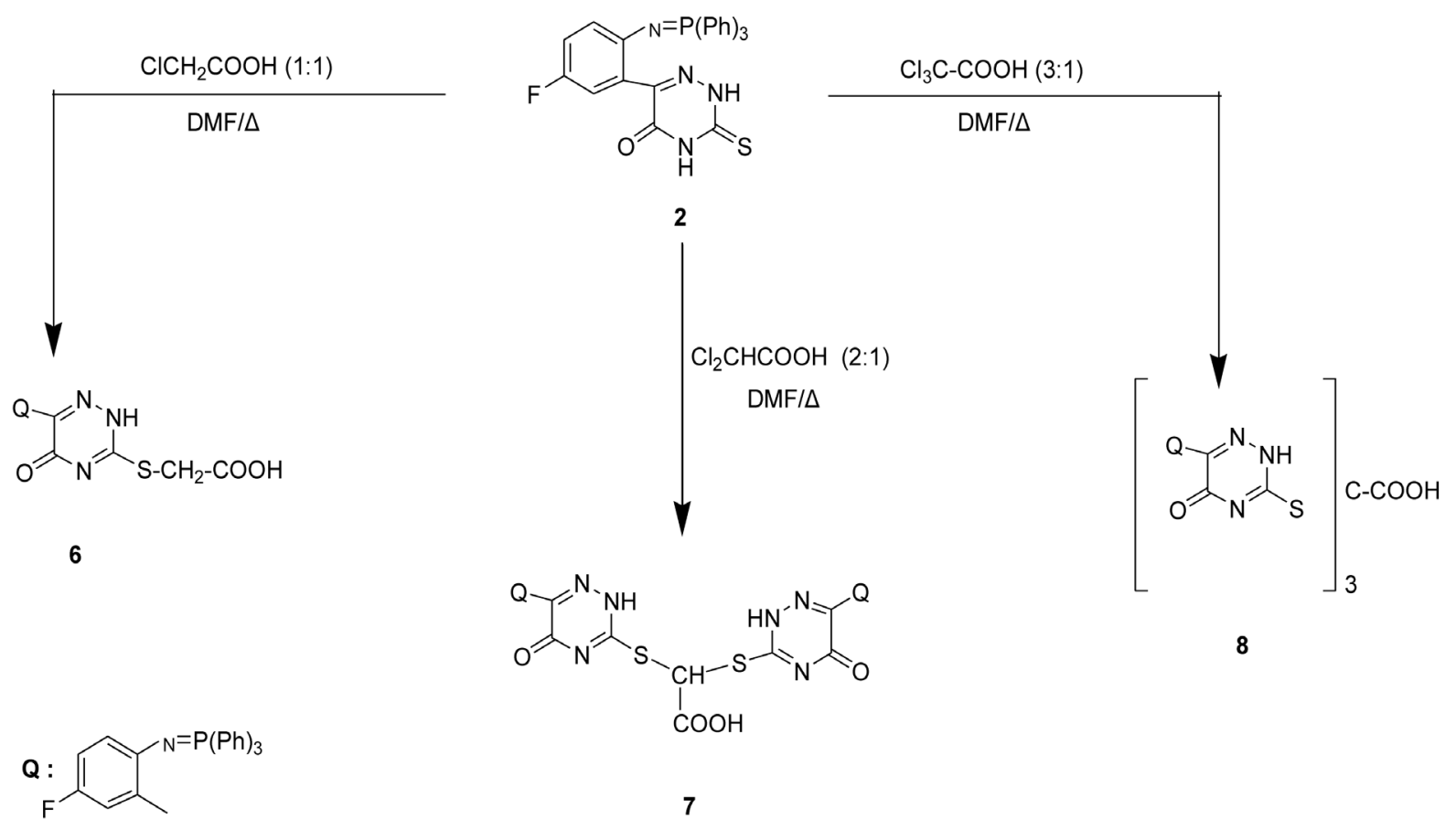

Scheme 3. Formation of compounds 6 - 8. 


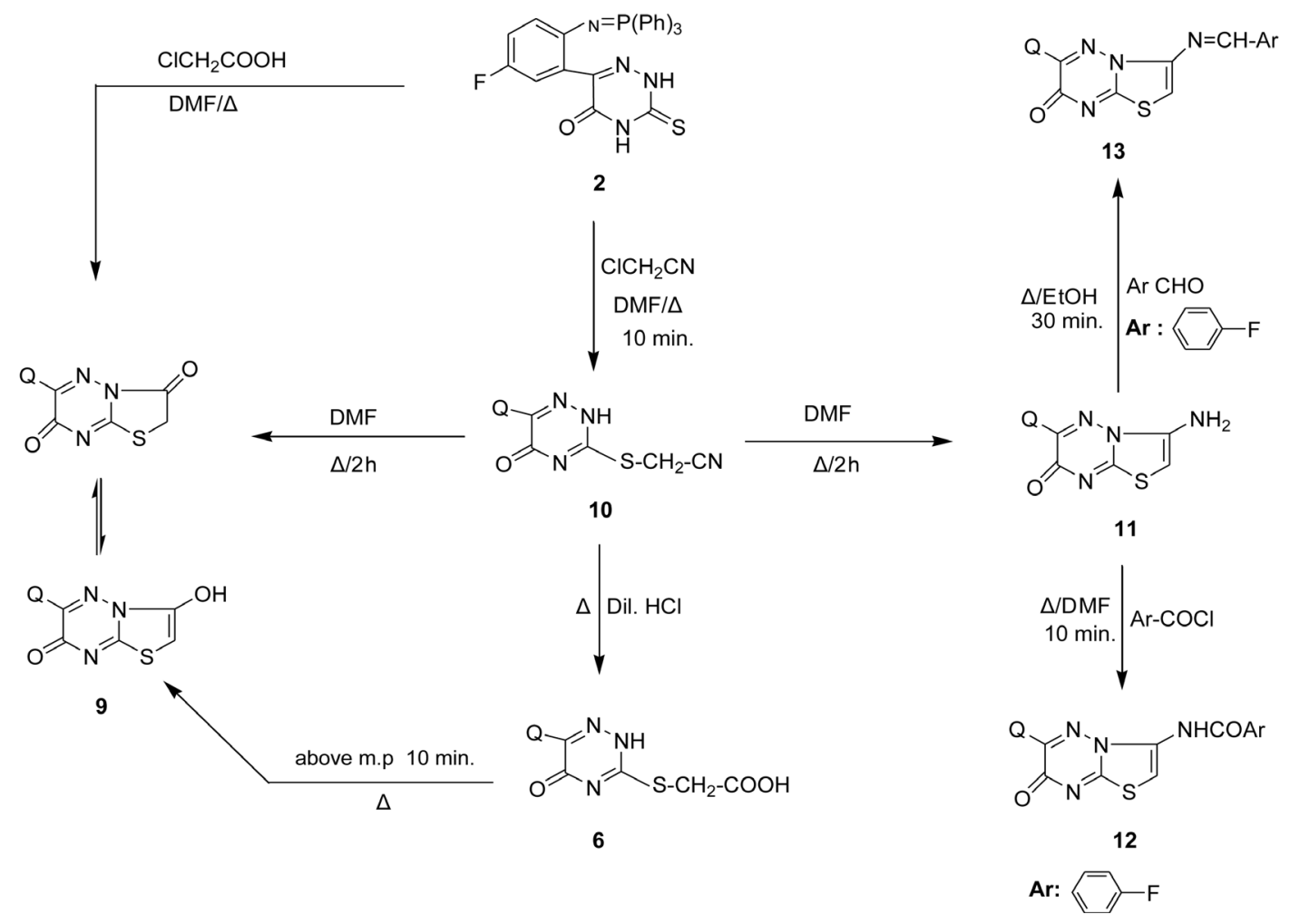

Scheme 4. Formation of compounds 9 - 13.

\subsection{Elucidation the Former Structures}

\subsubsection{UV Spectra}

The electronic conjugated molecule of compound 2 exhibited $\lambda_{\max }$ at $310 \mathrm{~nm}$ while that of compounds 3 (363), 5a (364), 8 (359) and 16 (323) nm. A higher absorption bands of new acyclic systems than that of $\mathbf{2}$ confirm that $\mathrm{N}$ - and S-substitution were formed. On the other hand, the absorption bands of fused heterobicycle compounds 9 (352), 17 (347) and 10 (321) nm is higher than the start 2 (310) nm. This is attributing to extension of heteroconjugation of heterobicylic systems through a type of cylization.

\subsubsection{IR Spectra}

The new compounds obtained recorded the absorption bands at $1380-1390,1250-1230 \mathrm{~cm}^{-1}$ due to presence of both $\mathrm{P}=\mathrm{N}$ and $\mathrm{C}-\mathrm{F}$ functional groups. Compounds 3-5 showed a lack of band at $3200-3100 \mathrm{~cm}^{-1}$ for $\mathrm{NH}=\mathrm{OH}$ of 1,2,4-triazinones, while that of compounds 6-8 and 10 recorded the absorption band at 3343 and $1643 \mathrm{~cm}^{-1}$ attributed to presence of ${ }^{4} \mathrm{NH} \&{ }^{5} \mathrm{C}=\mathrm{O}$ of 1,2,4-triazinone. Only compounds 9-14 showed a lack of the absorption bands at $1200-1100 \mathrm{~cm}^{-1}$ for $\mathrm{C}=\mathrm{S}$, which confirm that heterocyclization. In addition to the compounds 6-9 \& 18, 20 exhibited a two absorption bands at $v 1700$ and $1665 \mathrm{~cm}^{-1}$ due to the presence of two carbonyl groups. Also, IR absorption spectra of compounds 3-8, 9-10 and $\mathbf{1 6}$ recorded the absorption bands at $v 2975$ and 2885 $\mathrm{cm}^{-1}$ attributed to aliphatic functional groups [1] [14] [15] [26].

\subsubsection{NMR Spectral Study}

1) ${ }^{1} \mathrm{H}$ NMR spectrum of $\mathbf{1}$ showed a resonated signals at $\delta 14.6,12.6$ and $10.9 \mathrm{ppm}$ for $3 \mathrm{NH}$ with $\delta 8.6-0.80$, 7.69 - 7.64, $7.41-7.31 \mathrm{ppm}$ for three aromatic protons, while that of 3 exhibited a signals at $\delta 5.24$ and 4.98 ppm attributed to two $\mathrm{OH}$ with $\delta 2.92-2.88,2.62-2.58 \mathrm{ppm}$ for two $\mathrm{CH}_{2}$ protons. Compounds 3, 4 and 5 showed a lack's of ${ }^{4} \mathrm{NH}$ and ${ }^{2} \mathrm{NH}$ of 1,2,4-triazine moiety, while that of 5 recorded additional signals at $\delta 1.9$ and $1.75 \mathrm{ppm}$ of two methyl groups of antipyrine moiety. ${ }^{1} \mathrm{H}$ NMR spectra of 6-8 recorded $\delta$ at $12.7,4.7 \mathrm{ppm}$ for NH and $\mathrm{OH}$ protons, while that of $\mathbf{9}$ showed a signal at $\delta 10.5$ and $8.5 \mathrm{ppm}$, attributed to $\mathrm{OH}$ and $\mathrm{CH}=$ of thiazole moiety. In addition to compound $\mathbf{1 0}$ recorded a signals at $\delta 13.90,2.59 \mathrm{ppm}$ for $\mathrm{NH}, \mathrm{CH}_{2}$ protons, while that of 


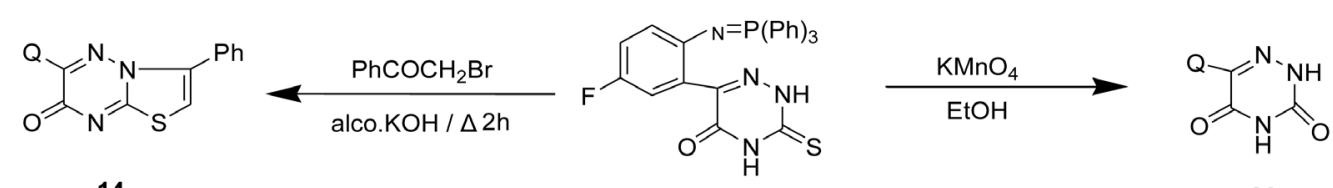

14

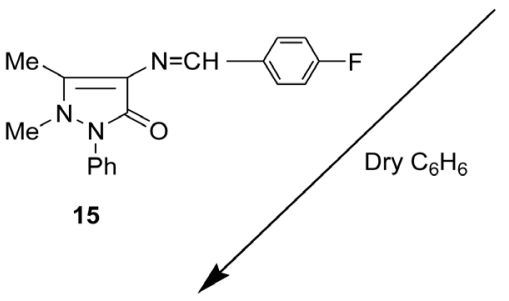

2

20

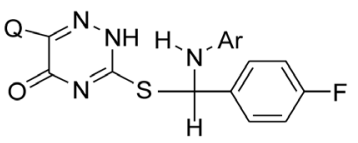

$\underbrace{\mathrm{CS}_{2}}_{2 \mathrm{DMF} / \Delta}$<smiles></smiles>

19

18

Ar:

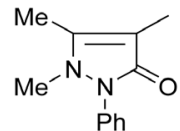

Q: ${ }^{\mathrm{N}=\mathrm{P}(\mathrm{Ph})_{3}}$

17

\section{Scheme 5. Formation of compounds 14 - 20.}

11 exhibited only signals at $\delta 8.01$ and $3.99 \mathrm{ppm}$ for $=\mathrm{CH}$ thiazole and amino-protons. Moreover ${ }^{1} \mathrm{H}$ NMR spectrum of 16 showed a signals at $\delta 12.76$ and 10.75 ppm for two $\mathrm{NH}$ of 1,2,4 trinazine while a lacks of these (2NH) protons of 17, with presence of $\mathrm{CH}$ proton of thiadiazine moiety at $\delta 9.68 \mathrm{ppm}$. ${ }^{1} \mathrm{H}$ NMR spectra of compounds 18 recorded the presence of $\delta$ at 14.55 and $12.79 \mathrm{ppm}$ attributed to $2 \mathrm{NH}$ of 1,2,4-triazine protons, while that of 19 exhibited a signals at $\delta 12.8$ and $10.7 \mathrm{p} \mathrm{pm}$ for $\mathrm{NH}$ and $\mathrm{CH} .\left(\mathrm{SO}_{2}-\mathrm{OH}\right)$ protons, with signals of aromatic protons. Finally, compound 20 exhibited $\delta$ at 12.73 and $10.82 \mathrm{ppm}$ attributed to $\mathrm{NH}$ and $\mathrm{OH}$ protons [14] [15] [19] [26].

2) ${ }^{13} \mathrm{C}$ NMR spectra of all the synthesized compounds showed a resonated signals at $\delta 180,165$ - 163, 140 138, $135-121$ and $112 \mathrm{p} \mathrm{pm}$ attributed to $\mathrm{C}=\mathrm{S}, \mathrm{C}=\mathrm{O}, \mathrm{C}=\mathrm{N}$, aromatic and $\mathrm{C}-\mathrm{F}$ carbons. Also, ${ }^{13} \mathrm{C}$ NMR spectra of compounds 3-6, 9 and 10 recorded signals at $\delta 39$ - 33 ppm for $\mathrm{CH}_{2}$ carbons. Only the compound 10 showed an additional signal at $\delta 112 \mathrm{p}$ pm for $\mathrm{C} \equiv \mathrm{N}$ carbon. Finally, ${ }^{13} \mathrm{C}$ NMR spectra of the entire compound exhibited a resonated signals at 77 - 75 ppm for C5-C6 of 1,2,4-triazine [27] (Figure 2).

3) ${ }^{19} \mathrm{~F}$ NMR spectral study recorded a signal at $\delta-126$ to $-125 \mathrm{ppm}$.

4) ${ }^{31} \mathrm{P}$ NMR spectral study exhibited a signal at $\delta 30-29 \mathrm{p} \mathrm{pm}$ attributed to $\mathrm{P}=\mathrm{N}$ [17].

\subsubsection{Mass Fragmentation Study}

Mass fragmentation pattern study of some selective synthesized compounds indicated that fused heterobiycyclic systems 11 have a more base peak, while that of acyclic structures 1and 16 have only base peak which indicate that their less stability. A higher stability of fused heterobicyclic systems is due to the delocalization of net charge over all the active centers (Figure 3 to Figure 5).

\section{Molluscicidal Activity}

Based upon the earlier work by Abdel-Rahman et al. [7] [16] on the synthesis of phosphono substituted-1,2,4- 


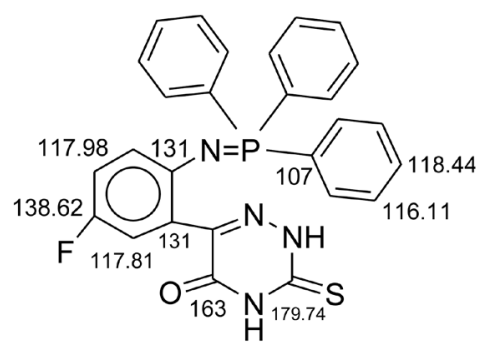

\section{Figure $2 .{ }^{13} \mathrm{C}$ NMR data of compound 2.}

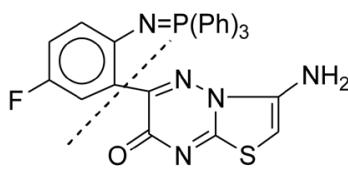

$11, M^{+} 537(3 \%)$

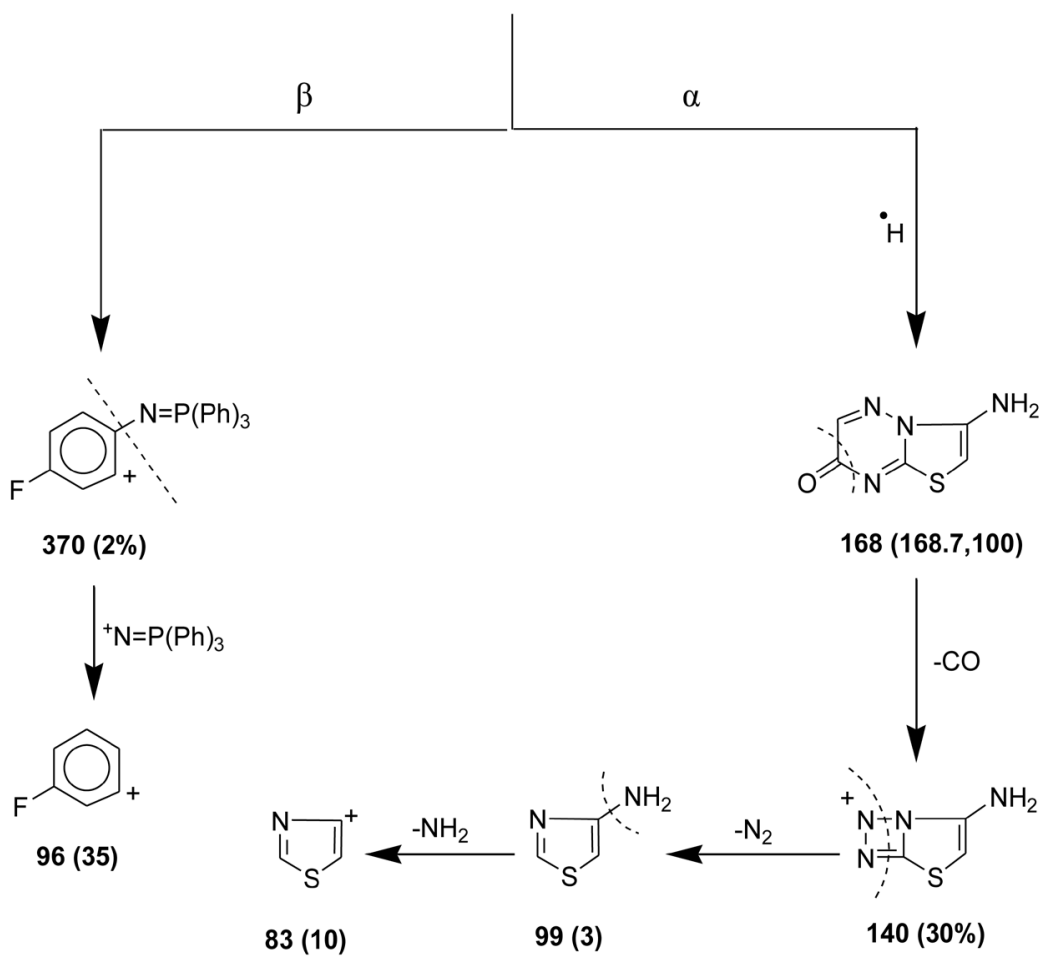

Figure 3. Mass fragmentation pattern of compound 11.

triazine derivative and their molluscicdal activities against Biomophalaria Alexandrina Snails responsible for Bilharziasis diseases, the prepared compounds were tested as killing of that snails ( shell in diameter 5 - 8). The intermediate host of sohistosomamausoni in Giza Govern state that was not treated with molluscicides. The snails were adapted to laboratory conditions for two weeks before being used in toxicity tests to be sure that the snails are strong and healthy. Snails were kept in plastic aguaria filled with de chlorinated tap water at room temperature $\left(25^{\circ} \mathrm{C}-27^{\circ} \mathrm{C}\right)$. Stock solution $\left(500 \mu \mathrm{g} \cdot \mathrm{ml}^{-1}\right)$ of the tested compounds were synthesized in the least volume of ethanol and completed of the least volume of ethanol and completed to the required volume with de chlorinated tap water on the basis of weight volume. A series of more diluted solutions were then prepared following the instructions given by WHO organization [28] [29]. The result given in (Table 1) revealed that the high activity towards snails in the following sequences:

$\mathbf{1 8}>\mathbf{2}>\mathbf{2 0}>\mathbf{3}>\mathbf{8}$ and $\mathbf{9}>\mathbf{1 0}>\mathbf{6}>\mathbf{1 7}>>\mathbf{5 a}$ and $\mathbf{5 b}>\mathbf{7}>\mathbf{1 4}$ at $100 \mathrm{ppm}$ in compared with Baylucide as 

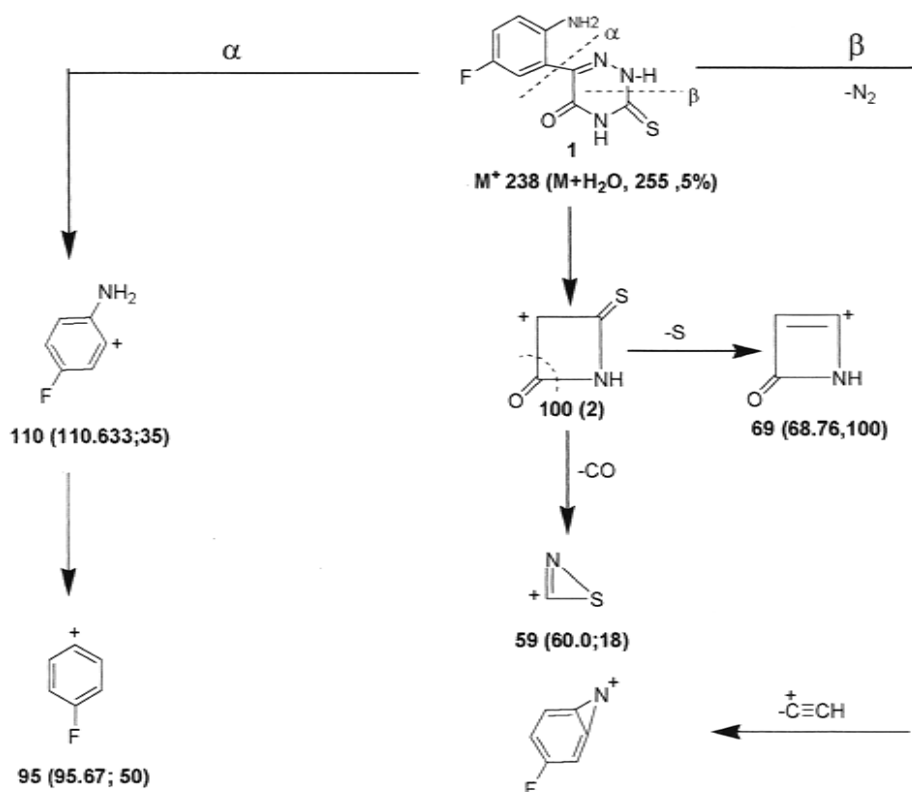

$M^{+} 238\left(M+H_{2} \mathrm{O}, 255,5 \%\right)$
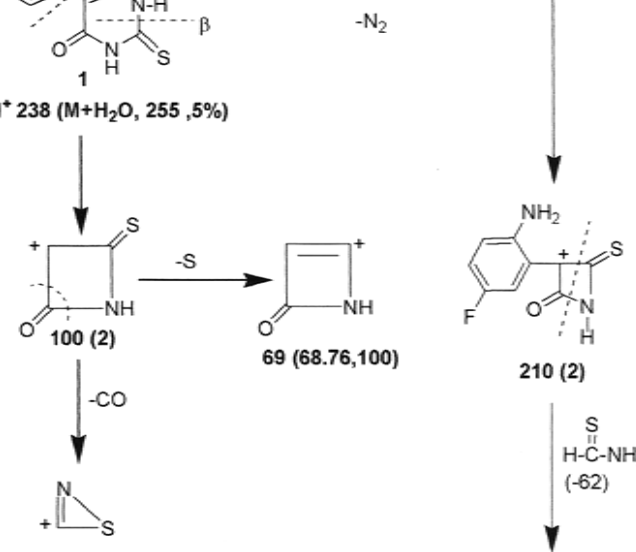

$210(2)$

$59(60.0 ; 18)$
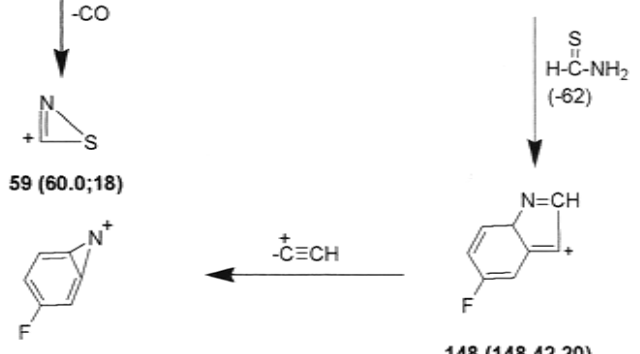

$108(20)$

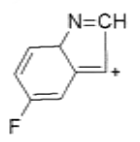

$148(148.42,20)$

Figure 4. Mass fragmentation pattern of compound 1.

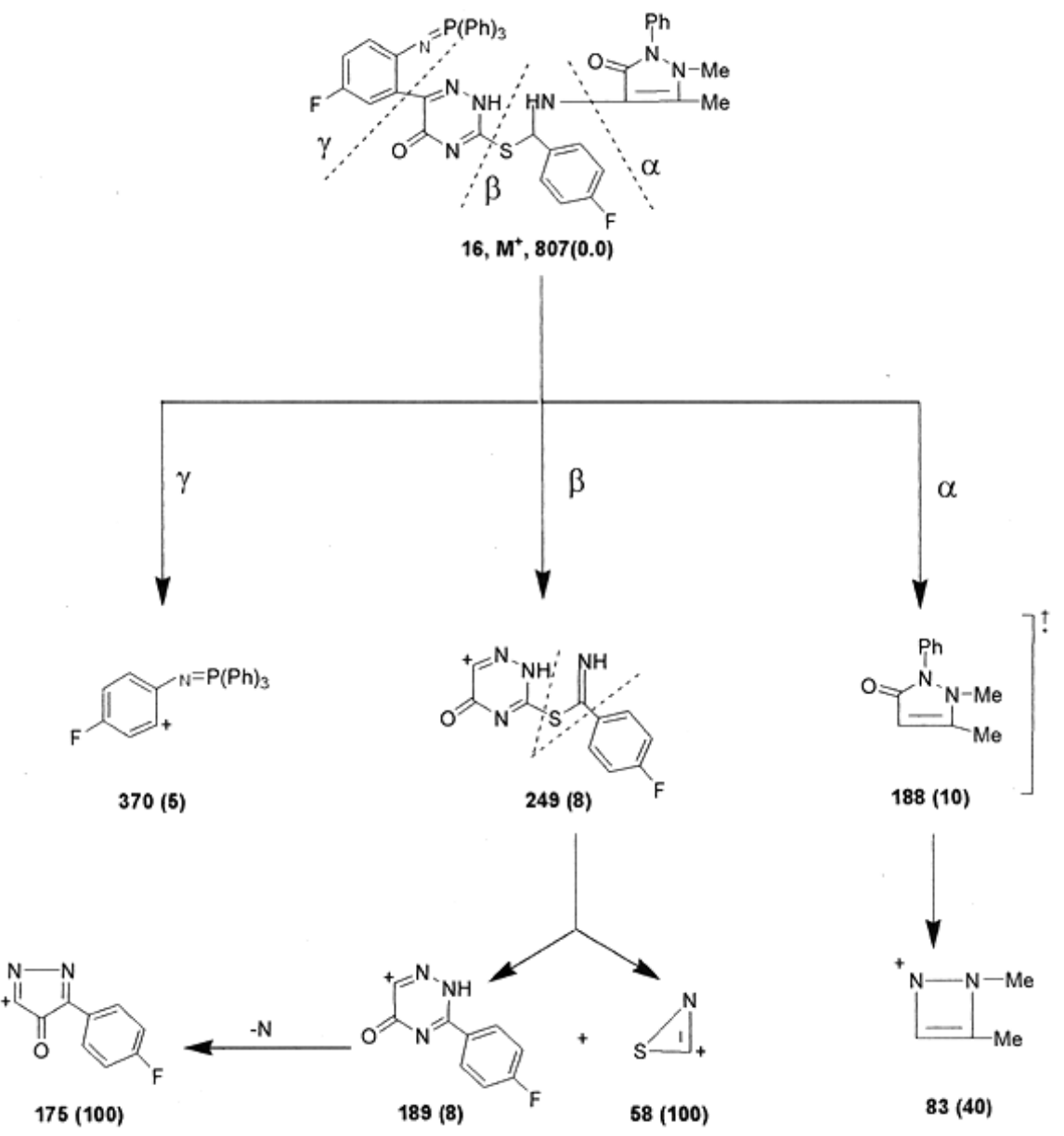

Figure 5. Mass fragmentation pattern of compound 16. 
Table 1 . The molluscicidal activity of the synthesized systems (2 - 20) mortality of snails various concentration (ppm).

\begin{tabular}{|c|c|c|c|}
\hline Comp. No. & 25 ppm & $50 \mathrm{ppm}$ & 100 ppm \\
\hline 2 & 30 & 60 & 80 \\
\hline 3 & 30 & 50 & 80 \\
\hline 4 & 20 & 30 & 60 \\
\hline $5 a$ & 20 & 40 & 70 \\
\hline $5 b$ & 10 & 20 & 50 \\
\hline 6 & 20 & 40 & 70 \\
\hline 7 & 20 & 30 & 50 \\
\hline 8 & 30 & 50 & 80 \\
\hline 9 & 30 & 50 & 70 \\
\hline 10 & 30 & 50 & 70 \\
\hline 12 & 10 & 20 & 30 \\
\hline $13 a$ & 10 & 20 & 30 \\
\hline $13 b$ & 10 & 20 & 30 \\
\hline 14 & 10 & 30 & 40 \\
\hline 16 & 10 & 20 & 30 \\
\hline 17 & 20 & 40 & 70 \\
\hline 18 & 40 & 60 & 90 \\
\hline 19 & 30 & 40 & 50 \\
\hline 20 & 30 & 60 & 80 \\
\hline \multicolumn{2}{|c|}{ Reference standard, Baylucide } & 100 & 100 \\
\hline
\end{tabular}

standard reference. In general, the strong effect of the compounds $2,3, \mathbf{8}, \mathbf{1 8}$ and 20 is due to presence both the $\mathrm{S}-\mathrm{S}, \mathrm{S}-\mathrm{H}$ and $\mathrm{O}-\mathrm{H}$ functional groups which agree with bio-oxidation-reduction processes. The moderate effect of the compounds 5a, 6, 9, 10 and $\mathbf{1 7}$ is attributed to thioether and cyclic sulfur nitrogen systems. Finally, the lethal effect of the compounds 4, 5b, 7, 11 and $\mathbf{1 4}$ may be to absence of $\mathrm{SH}$ and/or OH of Mannich base and for thiazolotriazine systems which led to the inhibition of delocalization electron-density over all the center of systems. Also, presence of hetero-elements (F, P, S, O) and N elements in corporated with 1,2,4-trinazines led to increases of electro-negativity, over all the molecular structure and enhance the electrostatic force and hydrophobic properties [17] [18] [31]-[33]. Thus, total electron-barrier of molecular distribution of the evaluated systems synthesized led to highly inhibition of the enzymatic effect on the living processes for the tested snails by causing break of a vital cyclic of that snails, and enhance the possibility killing of these snails. QSAR study of the obtained resulted from (Table 1), and based on the introduction of P, S and F in the synthesized 1,2,4-triazines, in compared with the mortality of tested snails, indicated that, increases of $\mathrm{P}$ and $\mathrm{S}$ percent \% led to increase of mortality, while, increase of F percentage \% led to decrease of mortality of snails. Also, very high electronegative of fluorine atom can modify the electronic distribution in the molecule affecting its absorption distribution and metabolism. In conclusion, 3-thioxo-1,2,4-triazine-5-ones bearing an P, S and F elements and their related S-alkyl derivatives, enhance the mortality of snails, which cause Bilharziasis Diseases than that their non-fluorinated and non-phosphinated systems. Also, increases of P and S percentage \% led to higher mortality of the tested snails, in hope to obtain more clean water from waste water. 


\section{Conclusion}

New fluorine substituted 6-(5'-fluoro-2'-triphenylphosphiniminophenyl) 3-thioxo-1,2,4-triazin-5 (2H, 4H) one (2) was obtained via Wittig's reaction of the corresponding 6-(5'-fluoro-2'-aminophenyl)-3-thioxo-1,2,4-triazinone (1). 3-thioxo-1,2,4-triazine-5-ones bearing an P, S and F elements and their related S-alkyl derivatives, enhance the mortality of snails, which cause Bilharziasis Diseases than that their non-fluorinated and non-phosphinated systems. Also, increases of $\mathrm{P}$ and $\mathrm{S}$ percentage \% led to higher mortality of the tested snails, in hope to obtain more clean water from waste water.

\section{Acknowledgements}

The authors are thankful to Prof. M. M. El-Sayed for helping in testing the molluscicidal activity in Theodor Bilharz Research institute, Giza, Egypt.

\section{References}

[1] Abdel-Rahman, R.M. and Ali, T.E. (2013) Synthesis and Biological Evaluation of Some New Polyfluorinated 4Thiazolidinone and $\alpha$-Aminophosphonic Acid Derivatives. Monatshefte fur Chemie, 144, 1243-1252. http://dx.doi.org/10.1007/s00706-013-0934-6

[2] Makki, M.S.T., Bakhotmah, D.A. and Abdel-Rahman, R.M. (2012) Highly Efficient Synthesis of Novel Fluorine Bearing Quinoline-4-Carboxylic Acid and the Related Compounds as Amylolytic Agents. International Journal of Organic Chemistry, 2, 49-55. http://dx.doi.org/10.4236/ijoc.2012.21009

[3] Makki, M.S.T., Bakhotmah, D.A., Abdel-Rahman, R.M. and El-Shahawy, M.S. (2012) Designing and Synthesis of New Fluorine Substituted Pyrimidine-Thion-5-Carbonitrles and the Related Derivatives as Photochemical Probe Agent for Inhibition of Vitiligo Disease. International Journal of Organic Chemistry, 2, 311-320. http://dx.doi.org/10.4236/ijoc.2012.223043

[4] Abdel-Rahman, R.M., Makki, M.S.T. and Bawazir, W.A. (2011) Synthesis of Some New Fluorine Heterocyclic Nitrogen Systems Derived from Sulfa Drugs as Photochemical Probe Agents for Inhibition of Vitiligo Disease Part I. E-Journal of Chemistry, 8, 405-414.

[5] Abdel-Rahman, R.M., Makki, M.S.T. and Bawazir, W.A. (2010) Syntheisis of Fluorine Heterocyclic Nitrogen Systems Derived from Sulfa Drugs as Photochemical Probe Agents for Inhibition of Vitiligo Disease Part II. E-Journal of Chemistry, 7, 593-5102.

[6] Abdel-Rahman, R.M. (2003) Synthesis of New Phosphaheterobicyclic Systems Containing 1,2,4-Triazine MoietyPart IX: Straight forward Synthesis of New Fluorine Bearing 5-Phospha-1,2,4 Triazine/1,2,4-Triazepine-3-Thiones; Part X: Synthesis of New Phosphaheterobicyclic Systems, Containing a 1,2,4-Triazine Moiety. Trends in Heterocyclic Chemistry, 8, 187-195.

[7] Ali, T.E., Abdel-Rahman, R.M., Hanafy, F.J. and El Edfawy, S.M. (2008) Synthesis and Molluscicidal Activity of Phosphorus-Containing Heterocyclic Compounds Derived from 5,6-Bis (4-brome phenyl)-3-hydrazino-1,2,4-triazine. Phosphorus, Sulfur, and Silicon, 183, 2565-2577.

[8] Abdel-Rahman, R.M., Ibrahim, M.A. and Ali, T.E. (2010) 1,2,4-Triazine Chemistry Part II, Synthetic Approaches for Phosphorus Containing 1,2,4-Triazine Derivatives. European Journal of Chemistry, 1, 388-396. http://dx.doi.org/10.5155/eurjchem.1.4.388-396.154

[9] Blakley, B., Brousseau, P., Fournier, M. and Voccia, I. (1999) Immunotoxicity of Pesticides. Toxicology Industrial Health, 15, 119-132. http://dx.doi.org/10.1177/074823379901500110

[10] Sengupta, A.K., Bajaj, O.P. and Agarwal, K.C. (1980) Synthesis and Insecticide Activity of N ${ }^{4}-$ Aryl-N $^{1}-\left(0.0-\right.$ Dial- $^{-}$ kylthiophosphoryl) Piperazines. Journal of the Indian Chemical Society, 57, 1170-1171.

[11] Du, Y.M., Tian, J., Liao, H., Bai, C.J., Yan, X.L. and Liu, G.D. (2009) Aluminum Tolerance and High Phosphorus Efficiency Helps Stylosanthes Better Adapt to Low-P Acid Soils. Annals of Botony, 103, 1239-1247. http://dx.doi.org/10.1093/aob/mcp074

[12] Abdel-Rahman, R.M. (2001) Role of Uncondensed 1,2,4-Triazine Derivatives as Biological Plant Protection Agents. Pharmazie, 56, 195-212.

[13] Abdel-Rahman, R.M. (2001) Role of Uncondensed 1,2,4-Triazine Compounds and Related Heterobicyclic Systems as Therapeutic Agents. Pharmazie, 56, 18-30.

[14] Abdel-Rahman, R.M. (1991) Synthesis and Anti Human Immune Virus Activity of Some New Fluorine Containing Substituted 3-Thixo-1,2-4-Triazin-5-Ones. Farmaco, 46, 379-389.

[15] Abdel-Rahman, R.M. (1992) Synthesis of Some New Fluorine Bearing Tri-Substituted 3-Thioxo-1,2,4-Triazine-5- 
Ones as Potential Anti Cencer Agent. Farmaco, 47, 319-326.

[16] Makki, M.S.T., Abdel-Rahman, R.M. and El-Shahawi, M.S. (2012) Synthesis and Voltammetric Study of Some New Macrocylis of Arsenic (III) in Wastewater and as Molluscicidal Agents against Biomophalaria Alexandrina Snails. Comptes Rendus Chimie, 15, 617-626.

[17] Basavaiah, D., Chandrashekar, V., Das, U. and Reddy, G.J. (2005) A Study toward Understanding the Role of a Phosphorus Stereogenic Center in (5S)-1,3-Diaza-2-Phospha-2-oxo-3-Phenylbicyclo(3.3.0)Octane Derivatives as Catalysts in the Borane-Mediated Asymmetric Reduction of Prochiral Ketones. Tetrahedron: Asymmetry, 16, 3955-3962. http://dx.doi.org/10.1016/j.tetasy.2005.10.038

[18] Ali, P., Pamakanth, P. and Meshram, J. (2010) Exploring Microwave Synthesis for Co-Ordination: Synthesis, Spectral Characterization and Comparative Study of Fluorine Substituted Transition Metal Complexes with Binuclear Core Derived from 4-Amino-2,3-Dimethyl-1-Phenyl-3-Pyrazolin-5-One. Journal of Coordination Chemistry, 63, 323-329. http://dx.doi.org/10.1080/00958970903305437

[19] Abdel-Rahman, R.M. (2000) Chemistry of Uncondensed 1,2,4-Triazines: Part II-Sulfur Containing 5-oxo-1,2,4-Triazin 3-yl Moiety. Phosphorus, Sulfur and Silicon, 166, 315-357. http://dx.doi.org/10.1080/10426500008076552

[20] Abdel-Rahman, R.M. and Islam, I.E. (1993) Synthesis and Reactions of Acetonitrile Derivatives Bearing a 5,6-Dipheny-1,2,4-Triazin-3-yl Moiety. Indian Journal of Chemistry, Section B, 32, 526-529.

[21] Abdel-Rahman, R.M., Seada, M., Fawzy, M.M. and El-Baz, I. (1993) Synthesis and Anti-Canceranti Human Immune Virus Activities of Some New Thioether Bearing a 1,2,4-Triazine-3-Hydrazones. Farmaco, 48, 397-406.

[22] EL-Gendy, Z., Morsy, J., Allimony, H.A., Abdel-Monem, W.R. and Abdel-Rahman, R.M. (2003) Synthesis of Heterobicyclic Nitrogen System Bearing the 1,2,4-Triazine Moiety as Anti-HIV and Anti-Cancer Drugs, Part II. Phosphorus, Sulfur and Silicon, 178, 2055-2071. http://dx.doi.org/10.1080/10426500390228738

[23] Abdel-Rahman, R.M. and El-Mahdy, K. (2012) Biological Evaluation of Pyramidpyrimidines as Multi-Targeted Small Molecule Inhibitors and Resistance Modifying Agents. Heterocycles, 85, 2391-2414. http://dx.doi.org/10.3987/REV-12-745

[24] Zaki, M.T., Abdel-Rahman, R.M. and El Sayed, A.Y. (1995) Use of Arylidenrhodanines for the Determination of Cu(II) $\mathrm{Hg}(\mathrm{II})$ and $\mathrm{CN}^{-}$. Analytica Chimica Acta, 307, 127-138. http://dx.doi.org/10.1016/0003-2670(95)00048-5

[25] Slawinski, J. and Gdaniec, M. (2005) Synthesis, Molecular Structure, and in Vitro Antitumor Activity of New 4Chlore-2-Mercaptobenzenesulfonamide Derivatives. European Journal of Medicinal Chemistry, 40, 377-389. http://dx.doi.org/10.1016/j.ejmech.2004.11.014

[26] Abdel-Rahman, R.M. and Abdel-Malik, N.S. (1990) Synthesis of Some New 3,6-Diheteroarryl-1,2,4-Triazine-5-Ones and Their Effect on Amylolytic Activity of Some Fungi. Pakistan Journal of Science and Industrial Research, 33, 142-147.

[27] Ebraheem, M.A., Abdel-Rahman, R.M., Abdel-Haleem, A.M., Ibrahim, S.S. and Allimony, H.A. (2008) Synthesis and Antifungal Activity of Novel Polyheterocyclic Compound Containing Fused 1,2,4-Triazine Moiety. Arkivoc, 21, $202-$ 213.

[28] WHO (1953) Expert Committee on Bilharziasis, 65, 33.

[29] WHO (1965) Snail Control Information of Bilharziasis Monograph Series. 50, 124.

[30] Smarti, B.E. (2001) Fluorine Substituent Effect (on Bioactivity). Journal of Fluorine Chemistry, 109, 3-11. http://dx.doi.org/10.1016/S0022-1139(01)00375-X

[31] Billard, T., Gille, S., Ferry, A., Bartelemy, A., Christophe, C. and Langlois, B.R. (2005) From Fluoral to Heterocycles: A Survey of Polyfluorinated Iminiums Chemistry. Journal of Fluorine Chemistry, 126, 189-196. http://dx.doi.org/10.1016/j.jfluchem.2004.08.007

[32] Isanbor, C. and O’Hagan, D. (2006) Fluorine in Medicinal Chemistry: A Review of Anti-Cancer Agents. Journal of Fluorine Chemistry, 127, 303-319. http://dx.doi.org/10.1016/j.jfluchem.2006.01.011

[33] Sandford, G. (2007) Elemental Fluorine in Organic Chemistry (1997-2006). Journal of Fluorine Chemistry, 128, 90104. http://dx.doi.org/10.1016/j.jfluchem.2006.10.019 
Scientific Research Publishing (SCIRP) is one of the largest Open Access journal publishers. It is currently publishing more than 200 open access, online, peer-reviewed journals covering a wide range of academic disciplines. SCIRP serves the worldwide academic communities and contributes to the progress and application of science with its publication.

Other selected journals from SCIRP are listed as below. Submit your manuscript to us via either submit@scirp.org or Online Submission Portal.
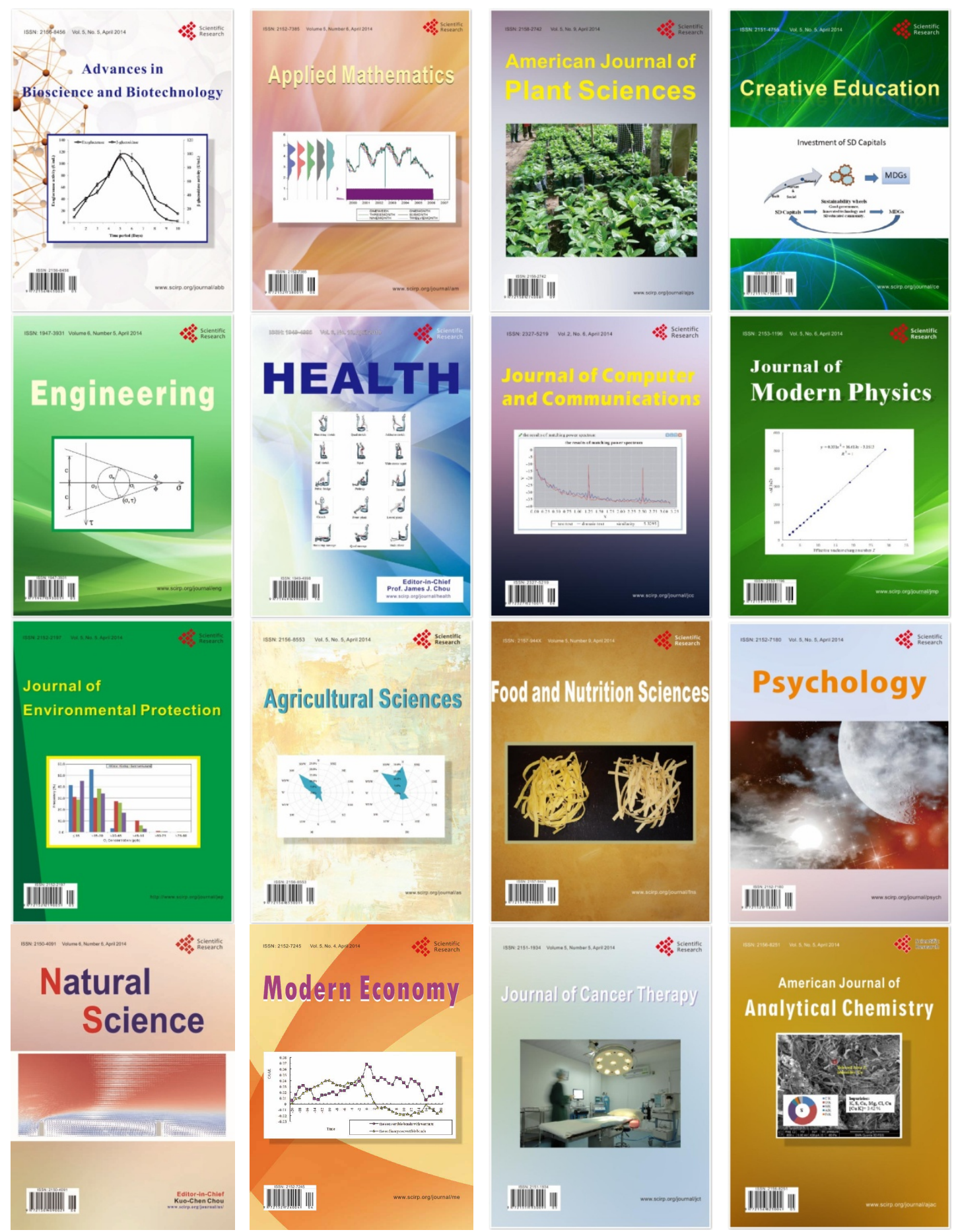